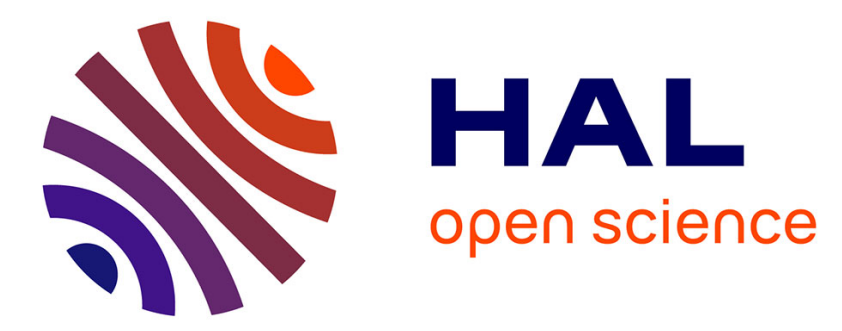

\title{
Influence de la protection cathodique sur le comportement électrochimique des couches de corrosion d'acier au carbone
}

Mai Tran Tron Long, Eliane Sutter, Bernard Tribollet

\section{To cite this version:}

Mai Tran Tron Long, Eliane Sutter, Bernard Tribollet. Influence de la protection cathodique sur le comportement électrochimique des couches de corrosion d'acier au carbone. Matériaux \& Techniques, 2013, 101 (5-6), pp.504. 10.1051/mattech/2013082 . hal-01023782

\section{HAL Id: hal-01023782 \\ https: / hal.sorbonne-universite.fr/hal-01023782}

Submitted on 15 Jul 2014

HAL is a multi-disciplinary open access archive for the deposit and dissemination of scientific research documents, whether they are published or not. The documents may come from teaching and research institutions in France or abroad, or from public or private research centers.
L'archive ouverte pluridisciplinaire $\mathbf{H A L}$, est destinée au dépôt et à la diffusion de documents scientifiques de niveau recherche, publiés ou non, émanant des établissements d'enseignement et de recherche français ou étrangers, des laboratoires publics ou privés. 


\title{
Influence de la protection cathodique sur le comportement électrochimique des couches de corrosion d'acier au carbone
}

\author{
T.T.M. Tran ${ }^{1}$, E. Sutter ${ }^{1}$ et B. Tribollet ${ }^{1}$
}

Reçu le 4 juillet 2013, accepté le 22 octubre 2013

\begin{abstract}
Résumé - Les propriétés électrochiniques de la couche de dépôts de corrosion formée ì la surface des coupons d'acier E24 immergés pendant plusieurs aunées dans l'eau de mer sous différentes condlitions de protection cathorlique ont été étudiées à l'aide de la spectroscopie d’impédance électrochimicque et de nesures globale ef locale de courant. Les résultats obtemus montrent que la conche de dépots dont le: comportement est déferminé par sa sous-couche interne est hétérogène, poreuse, conductrice et protectrice. La protection catledique favorise la formation de dépôts plus protecteurs. Une fois la conche formée, son pouvoir protecteur est pen infhencé par des changenents du mode de protection survenus ultérieurement. lorsque cenx-ci sont de courte durée.
\end{abstract}

\begin{abstract}
Mots clés : Acier an carbone / comrosion marine / protection catherlique / spectroscoppe d’inpédance clectrochinuigue / mesture de comrant local

Abstract - Influence of cathodic protection on the electrochemical behaviour of the carbon steel corrosion layers. The electrochemical properties of the corrosion deposit layer formed on the surface of the E24 steel coupons immersed for several years in seawater under difierent cathodic protection

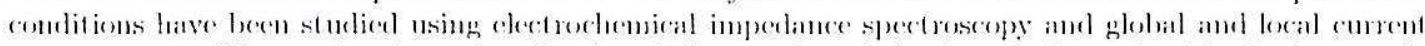

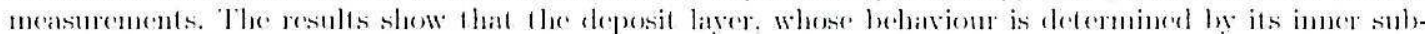

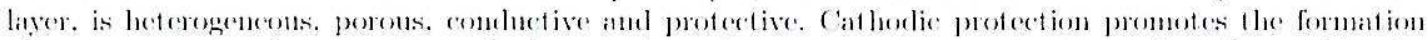

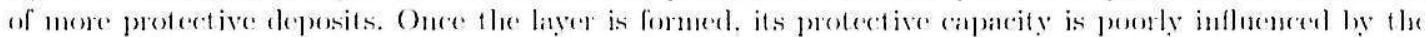
changes in the protertion conclitions which orem later. When they are of shent durat ion.
\end{abstract}

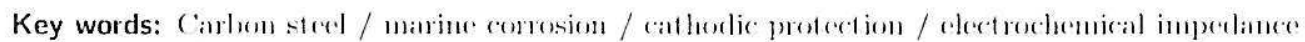

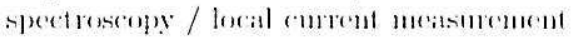

\section{Introduction}

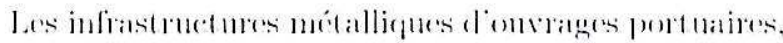

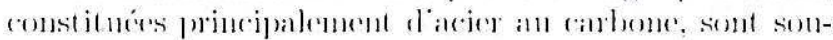
mises it des comblitions conviromonomentales sevieres an

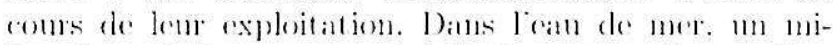

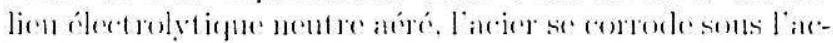

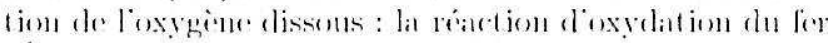

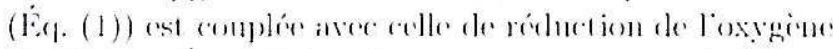

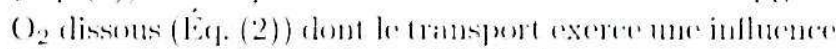

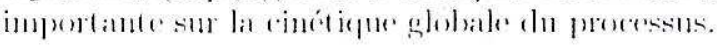

$$
\begin{aligned}
& \mathrm{ra} \rightarrow \mathrm{Ha}^{2}+2 \mathrm{C} \\
& 211.00+(0)+40 \rightarrow 10 H 1 \text {. }
\end{aligned}
$$

Le processus de comosion est remelu plus complexe par la

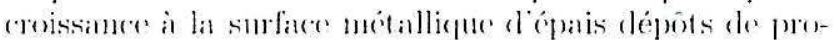

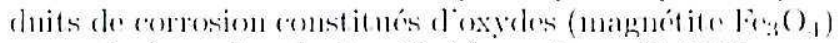

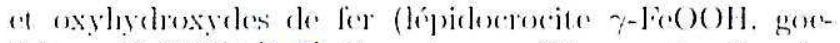
thite a-Fo(O) [1.2]. Dans les condlitions natmelles. les

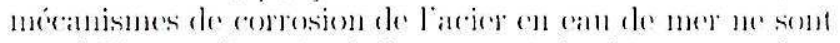

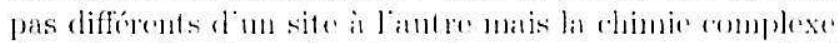

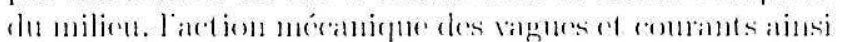

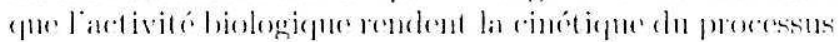

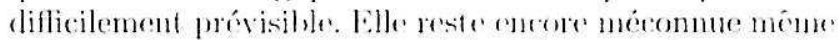

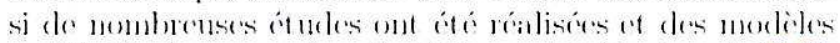

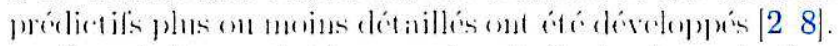

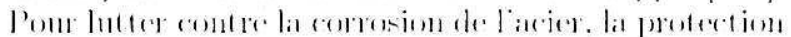

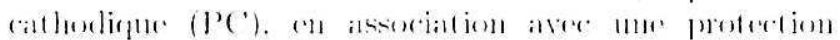

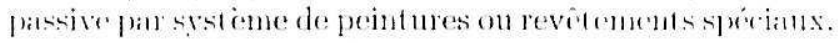

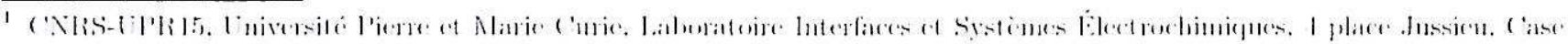

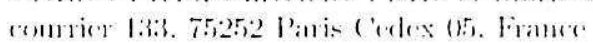

mai. tran_trong-long@upmc . $\mathrm{r}$
} 


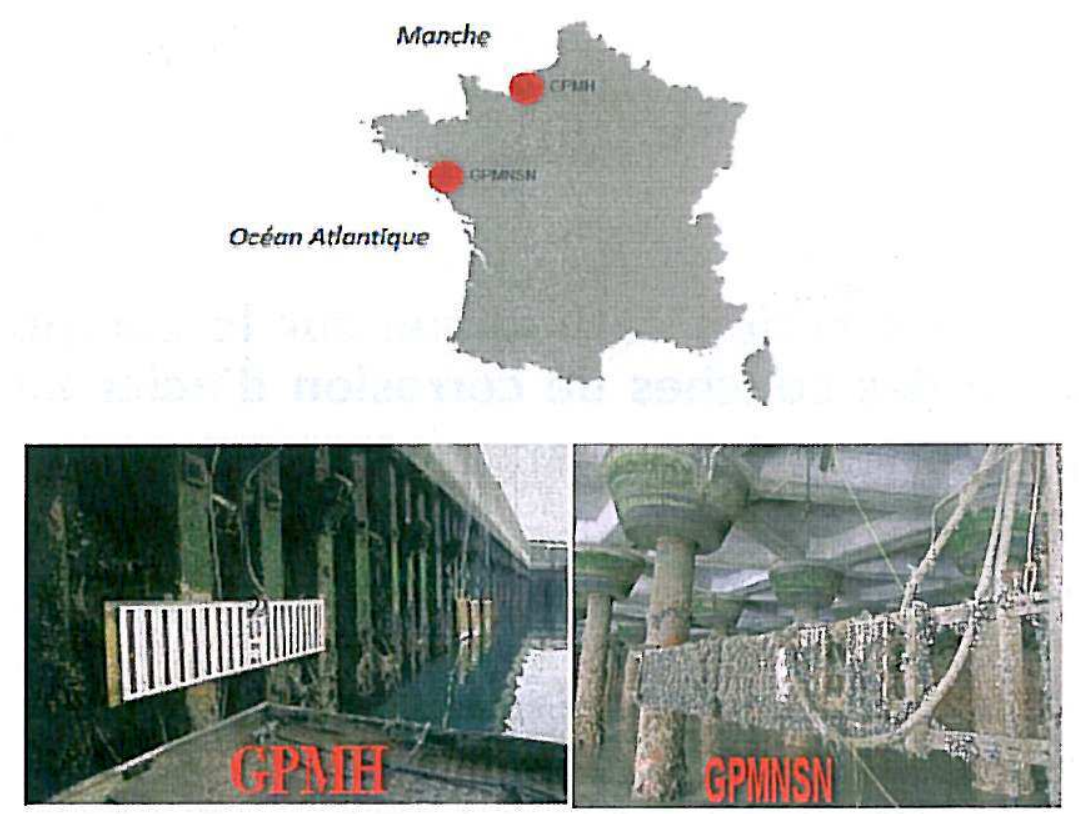

Fig. 1. Portiques d'échantillons exposés dans des sites de GPMII (avant exposition) et GPNNSN (an coms de l'exposition). Fig. 1. Sample porticos erposed in siles GPMHI (before ezposure) and GiPNNSN (during orposure).

est trò largenent utilisée on milien portuaire. La techniepue consiste à éviter on réluire la comeston du métal

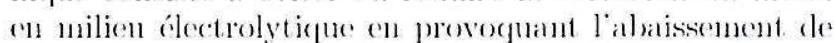
son potent ied édectrochinique jusqu à un niveau suffisant. Cot alaissement de potentiel est assuré par le couplage

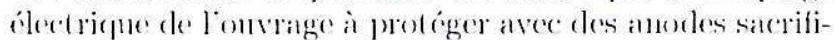
rielles en matrian moins noble (systeme gilvanique) on pat lönjection diun eomant blectriefue rontinn (systime

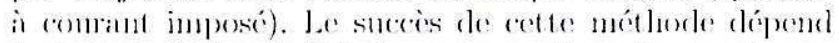
fortement de la gualite de sal conceptions. de sal mise ren

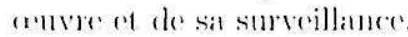

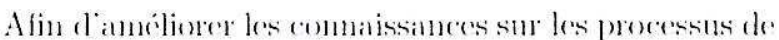

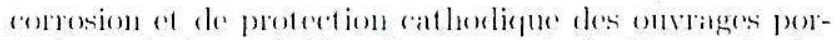
tuaires, ainsi que datfiner les capacités de diagnost ire des

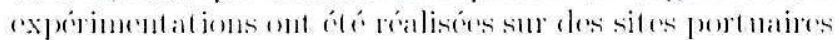

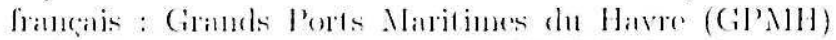

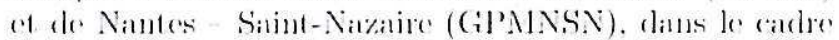
du pogrimme ('EMAR-P'C (C'orrosion Elentrochimique Maritime P'rotertion ('atleorligne).

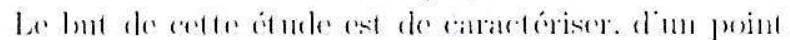

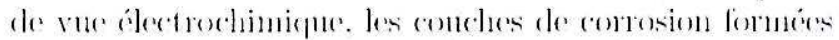

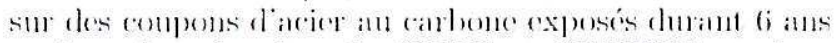

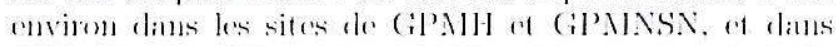

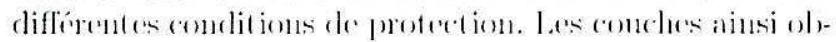

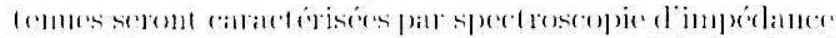

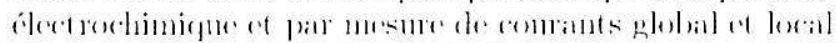

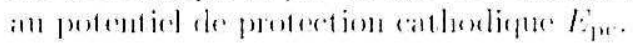

\section{Procédures expérimentales}

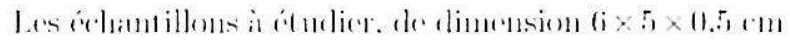

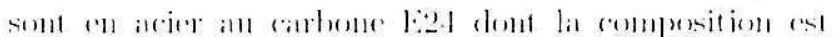

Tableau 1. Composition dhinicgue en \% matsigue de l'acier F.4 (domuces fournies par (TEMAR-PC).

Table I. Chemiral composition in why of the E2S sted (data provided by (EM/AR-P(:).

\begin{tabular}{|c|c|}
\hline É|е́ment & 'Теиени (\% (1) masse) \\
\hline Cimbone & 0,120 \\
\hline Mangancese & (1). .380 \\
\hline P'sesplose & $(1),(1) \mid 0$ \\
\hline Soufre & 0.001 .1 \\
\hline Silic·innı & (1).(1017 \\
\hline ('uiver & 0.. 1017 \\
\hline ('hrome & (1).11)7 \\
\hline Nirkel & (1).112:3 \\
\hline Almuinimm & $0.113 \overline{-}$ \\
\hline Molybline & 0,0011 \\
\hline $\mathrm{Fec}$ & bislanee \\
\hline
\end{tabular}

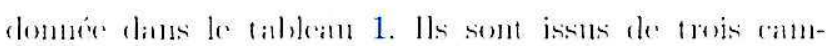

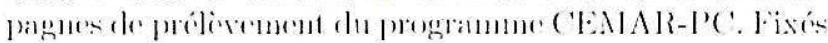
sull des portiques (lög. 1), les rompons sont restes int-

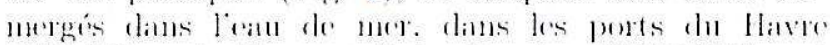

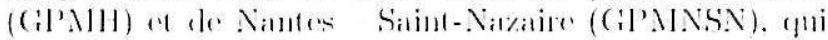
sont representatifs des stes portuaires strictement matri-

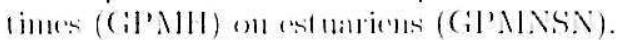

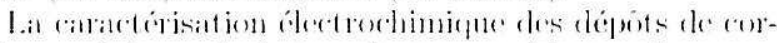

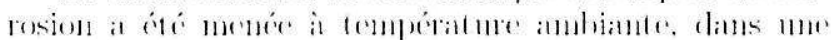

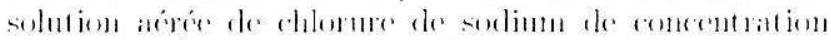

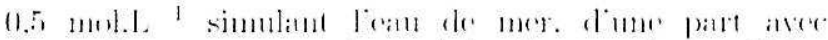

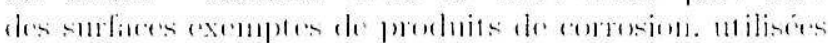

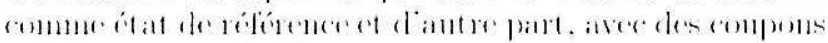

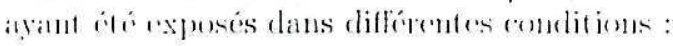

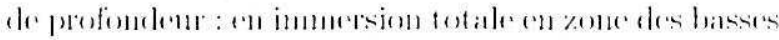

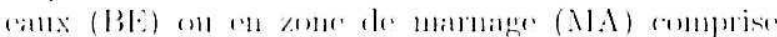



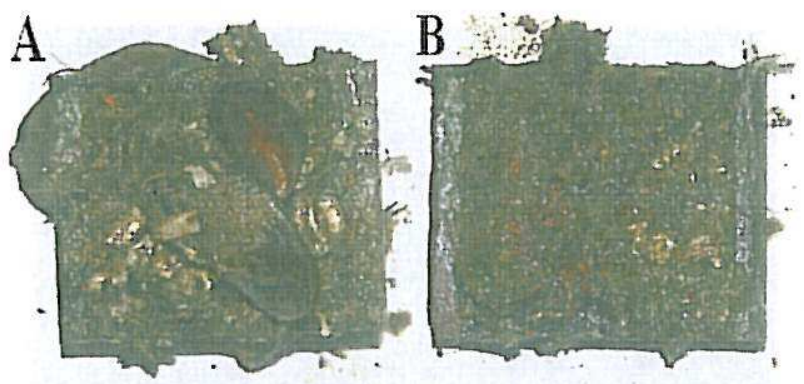

Fig. 2. Aspect de surface d'un coupon d'acier exposé dans un site de GPNH (BE, $\mathrm{PC}$ ) avant $(\mathrm{A})$ et après $(\mathrm{B})$ enlèvenent. des coquillages peu alliérents.

Fig. 2. Surface appearance of a sted coupon exposed in site GPMH (BE, PC) before (A) and after (B) removal of poorly adherent steel shells.

entre les niveaux de leau à marée basse et à marée haute aux plus forts coefficients;

- d'orientation : face d'exposition coté mer ou coté structure:

- de mode de protection : sans protection, sons PC permanente, avec mise sous PC d'échantillons nom protégés initialenent, avec rupture temporaire ou après arrêt de $\mathrm{PC}$.

Leséchantillons deacier poli ont été obtenus à partir de coupons corrodés après polissage à lanicle de papiers alurisifs an carbure de silicimm SiC de gramulométrie 1200. lese dépôts formés à la surfice des compons contiement sonvent. en plus des produits de corrosion, des micro of macro-organismes (moules voire huitres). C'es rleruiers ont

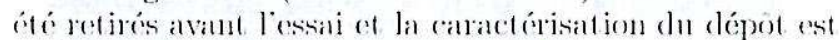
réalisé sur la coutle restante (Fig. 2).

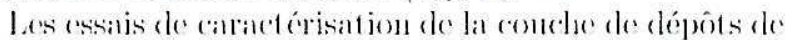

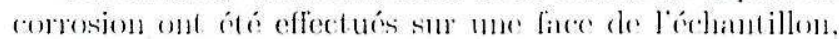

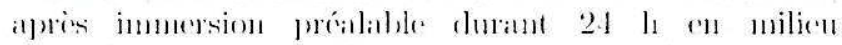

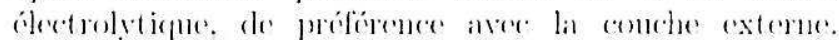

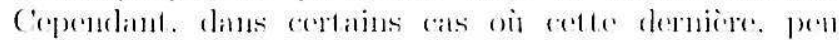

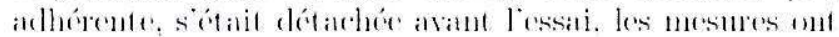

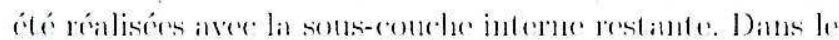

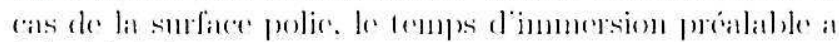
ale redult is :30 mint.

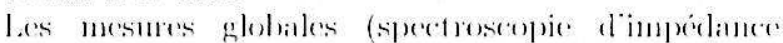

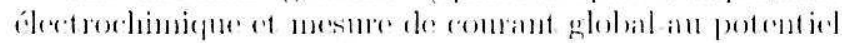

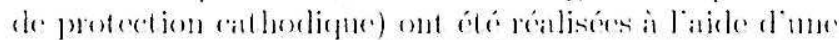

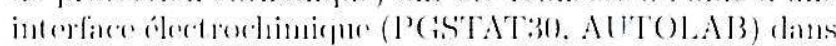

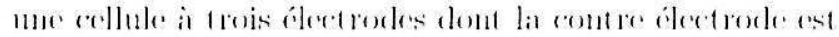

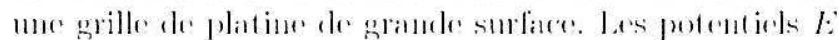

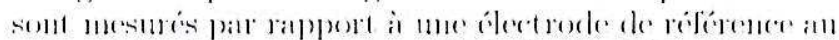

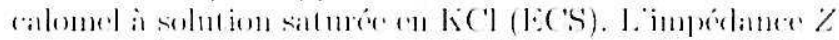

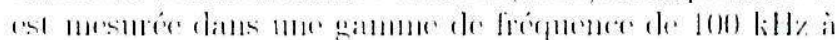

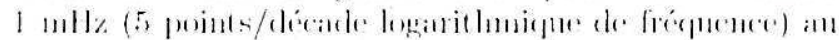

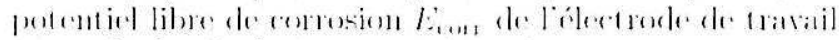

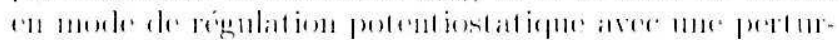

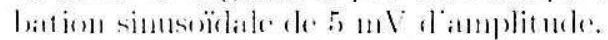

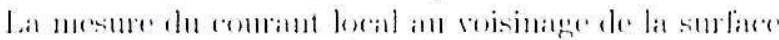

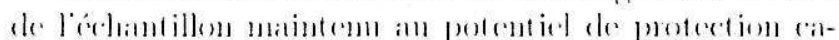

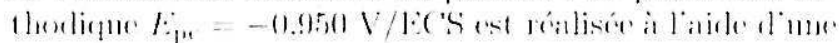
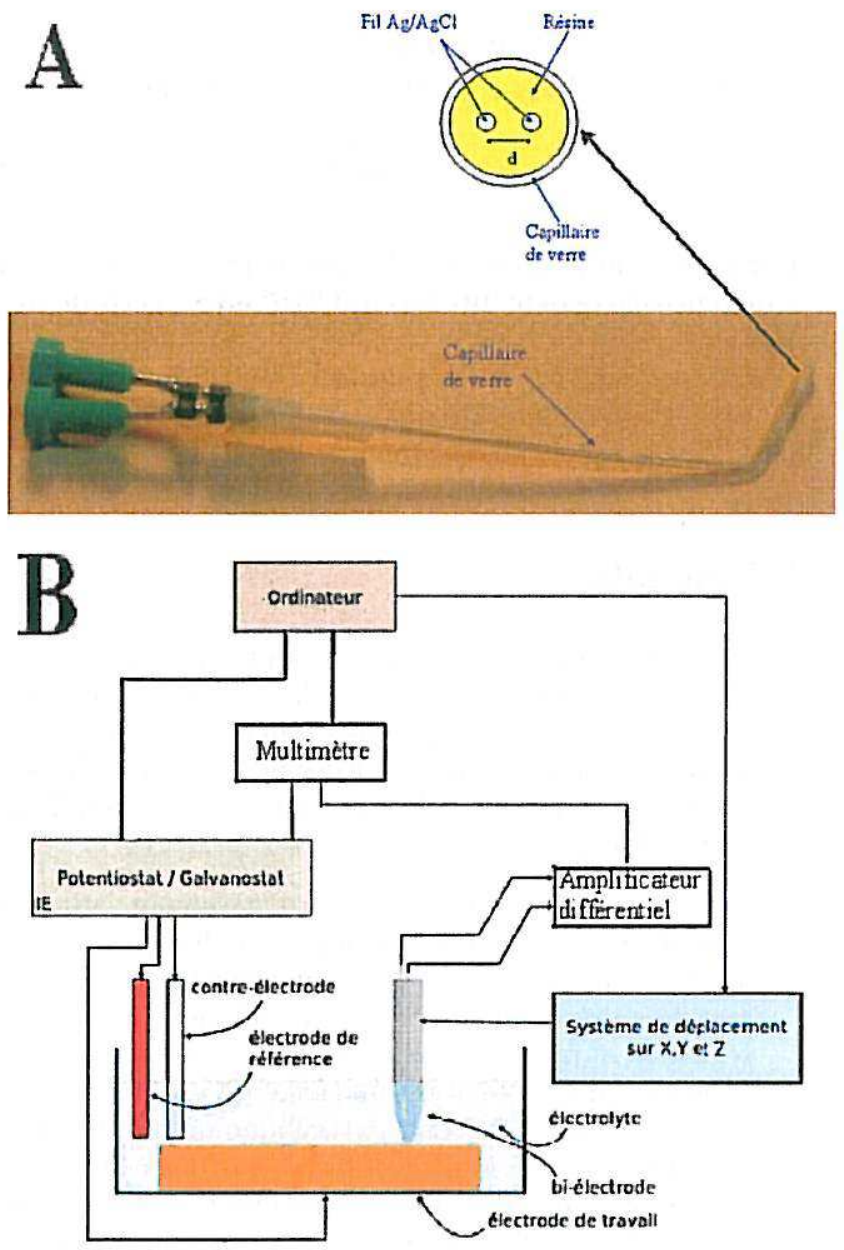

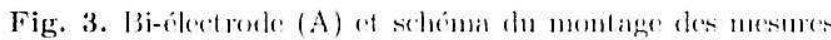
licioles (13).

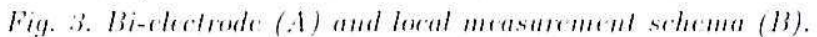

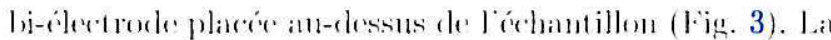
difference de potentiol lorial $\left(\Delta E_{\text {lecal }}\right)$ est mesmere entle

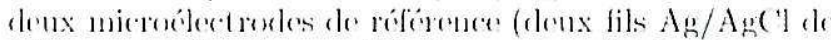

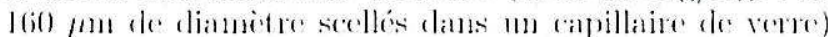

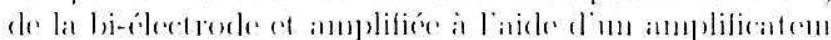
diflérentiel (AM502 I)ifferential Ampl, 'TEK'lRONIX).

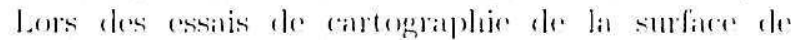

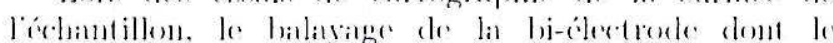

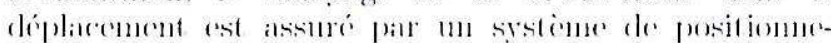

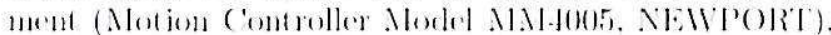
a bé effectur sur tomte la zome de surfire de travail

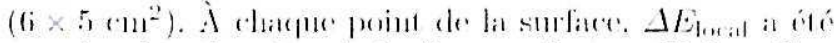

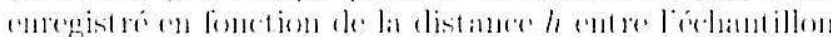

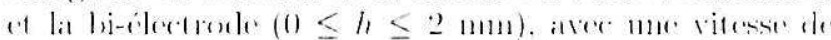

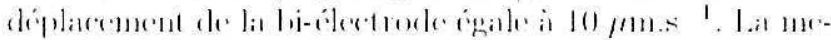

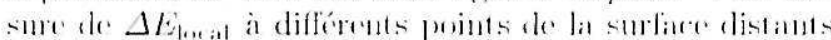

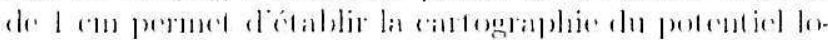

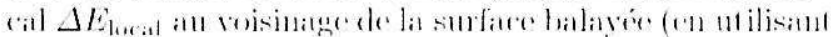

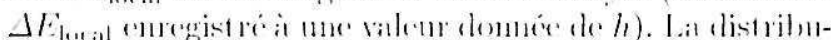

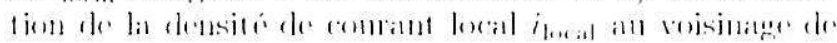


la surface de l'échantillon est obtenue à partir de celle du potentiel local $\Delta E_{\text {local }}$ en appliquant la loi d'Ohm :

$$
i_{\text {locial }}=\frac{\sigma \Delta E_{\text {locist }}}{d}
$$

où $\sigma$ est la conductivité de l'électrolyte (S.cm $\left.{ }^{-1}\right)$ et $d(\mathrm{~cm})$ la distance entre deux fils $\mathrm{Ag} / \mathrm{AgCl}$ de la bi-électrode $(d=$ $0,05 \mathrm{~cm})$.

La conductivité de la solution $\mathrm{NaCl}(0,5 \mathrm{M}$, mesurée a laide d'un conductinètre (Mettler Toledo, norlèle SevenEasy), est de $40,65 \mathrm{mS} . \mathrm{cm}^{-1}$

\section{Résultats}

Liexamen visuel des coupons montre que les conches de dépots de corrosion formées à la surface des coupons exposés coté mer ne sont pas très différentes de colles des coupons placés côté structure. Elles peuvent être classies, selon leur aspect et leur adhérence à la surface métallique. en deux catégories :

- Le ler type de conche est redativenent adlérent (Fig. 4. A). Les conches de dépots menferment, on plus des produits de corrosion, me quantité importante de matieres organiques (copullagess, algues...). Elles se rencont rent plus sonvent sur des échatut illons ayant été

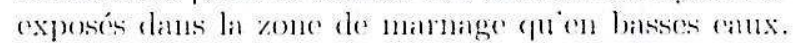

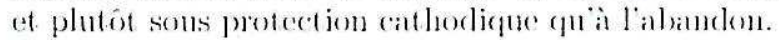

- be 2 eme type de couche se forme sur des édhantillons

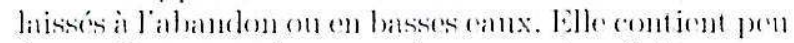

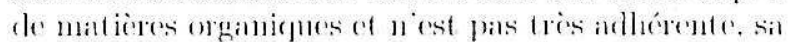

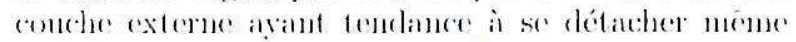
avint toute manipulat ion (1.ig. 4, B).

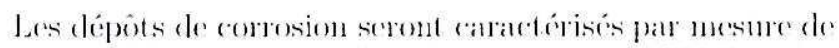

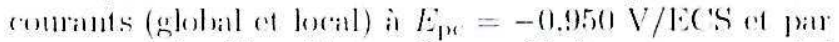

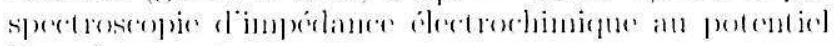
lihere de comosions.

\subsection{Mesure de courant global au potentiel de protection cathodique}

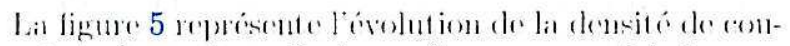

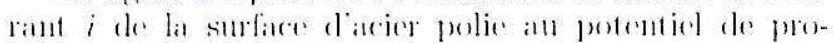

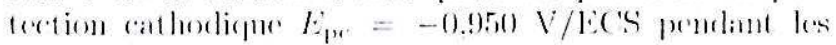

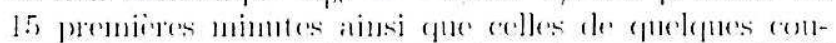

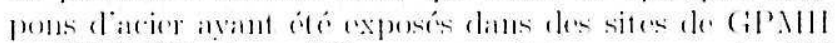

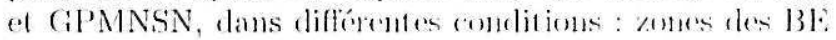

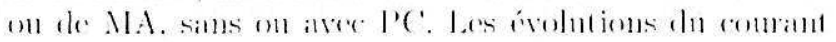

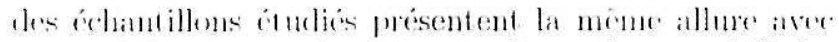

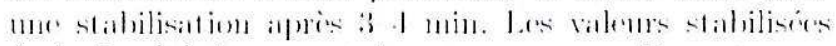

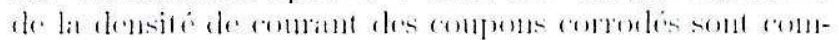

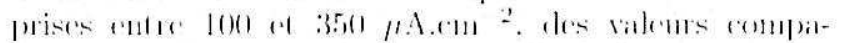

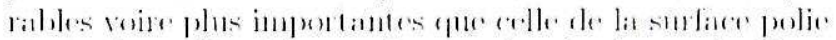

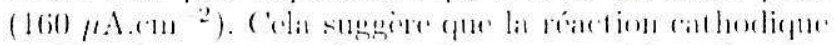

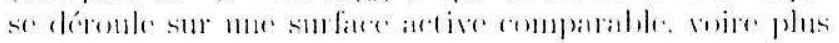

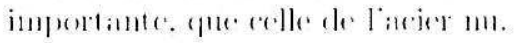
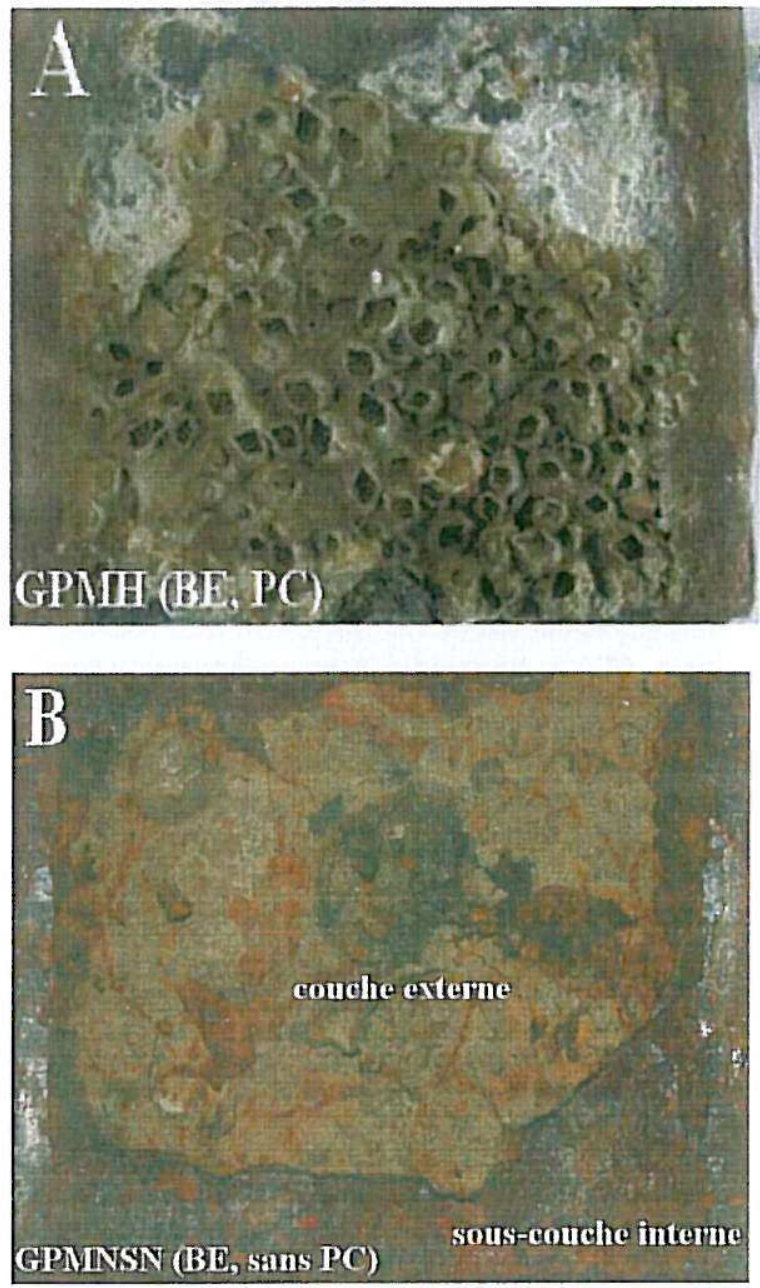

Fig. 4. Aspeed de la couche de dépots de comosion formés is

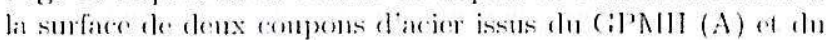
(BPMNSN (B).

Fig. \&. Appouraner of the comrosion deposil layer formed an

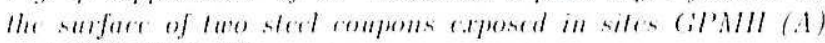
amil CiPMINSSN (B).

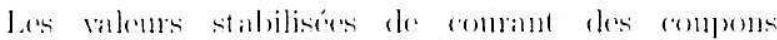

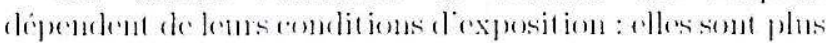

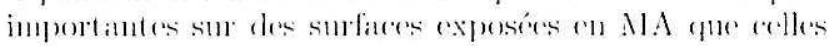

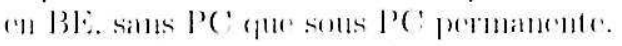

\subsection{Mesure de courant local au potentiel de protection cathodique}

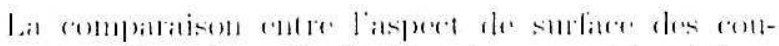

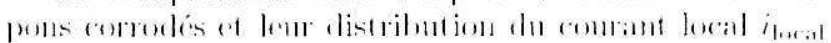

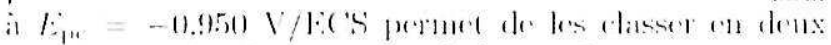

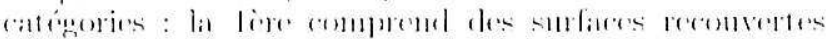
de porluits de (o)

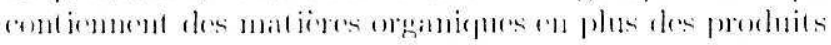
de corrosions. 
T.T.M. Tran et al. : Matériaux \& Techniques 101, 504 (2013)

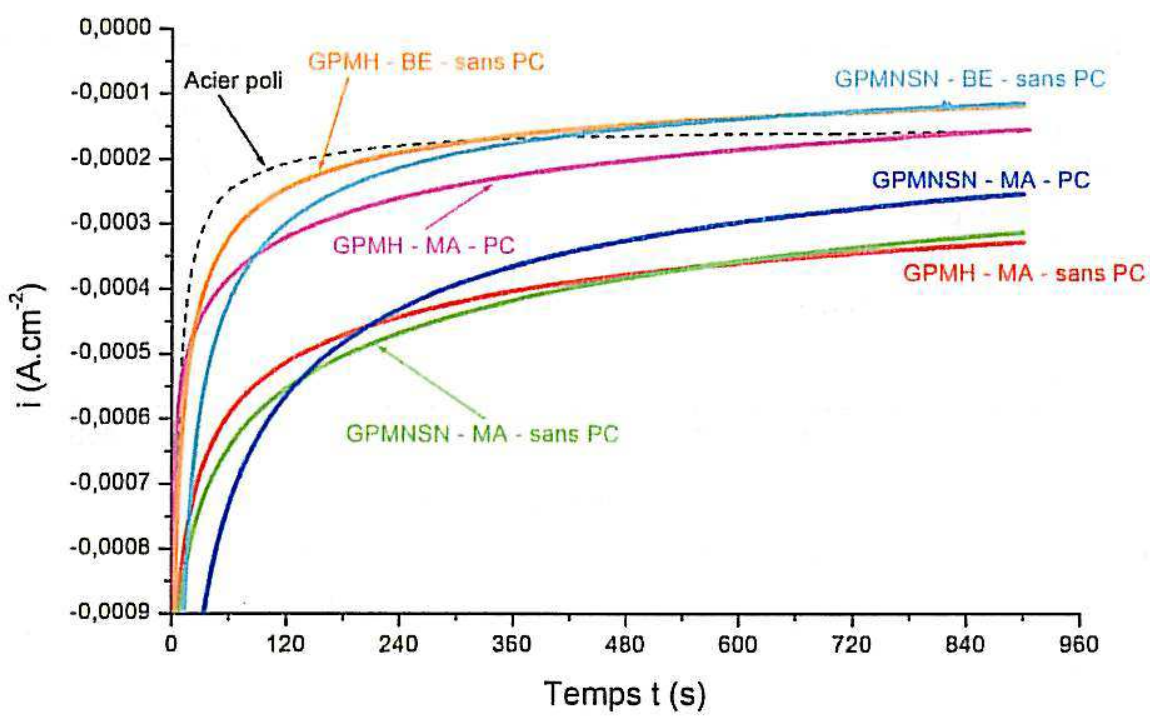

Fig. 5. Évolution de comant $i$ enregistrée an coms des essais potentiostatiques en solution NaCl $0.5 \mathrm{M}$ aérée à $E_{11}=$ -0.950 V/ECS sur les surfaces polie et corrodée de quelques coupons d'acier issus de GPMH et GPMNSN.

Fig. 5. Current enolution reconded during potculiostalic tests in $0.5 \mathrm{M} \mathrm{NaCl} \mathrm{aeraled} \mathrm{solution} \mathrm{at} E_{10}=-0.950 \mathrm{~V} / S C E$. of $1 / \mathrm{he}$ polished surface and of a frew sted coupons exposed in sites GPMIII and GPMNSN.
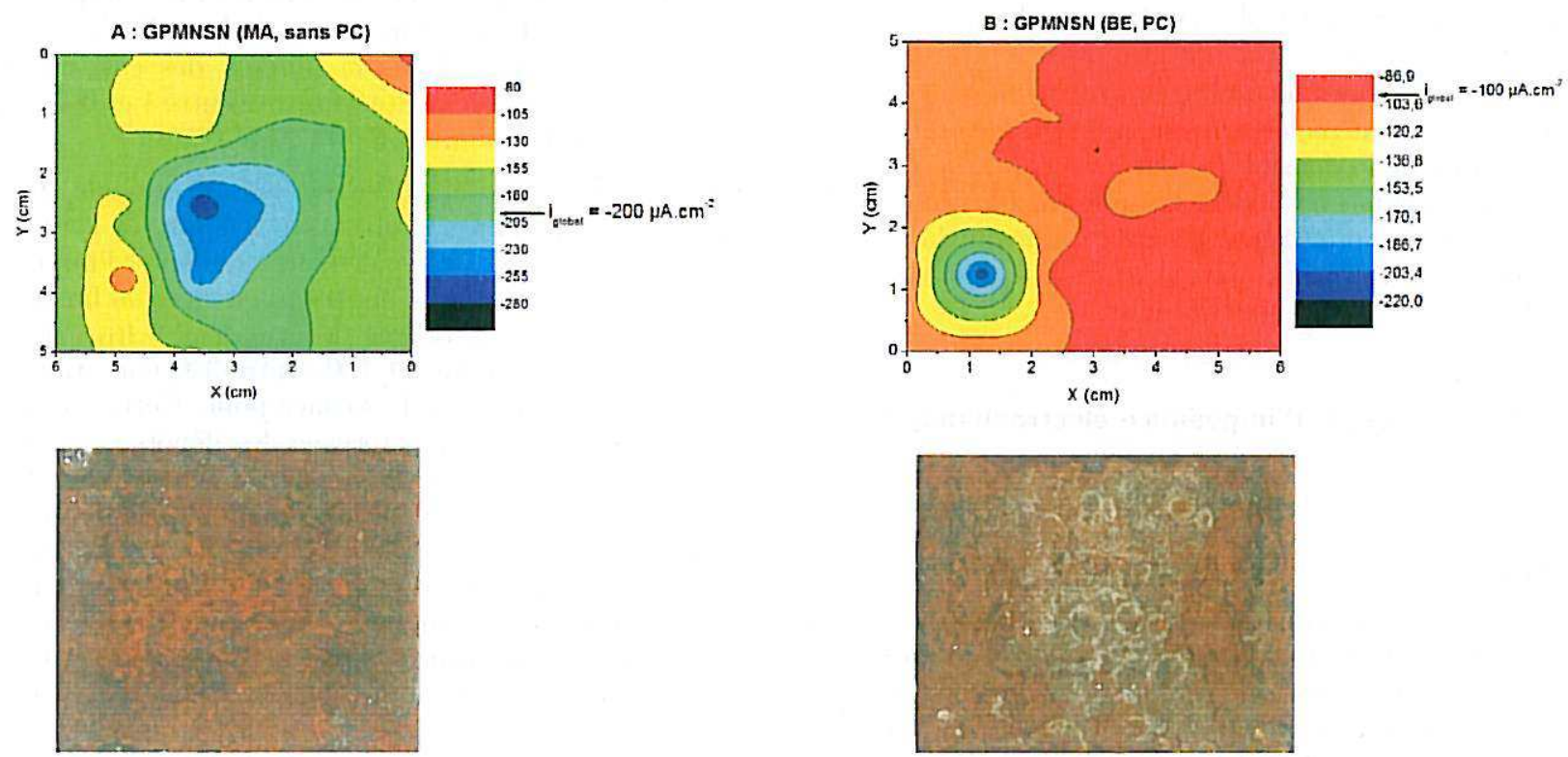

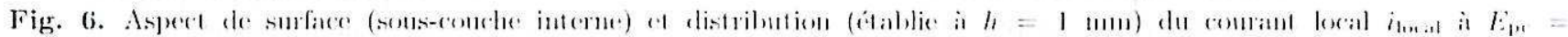

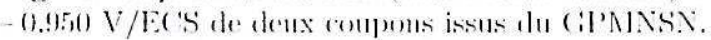

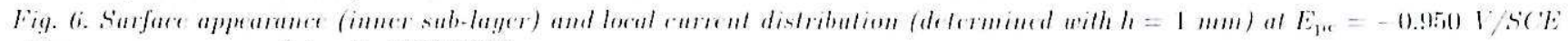

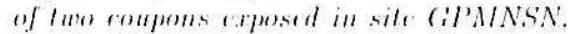

\subsubsection{Surfaces recouvertes de produits de corrosion (catégorie 1)}

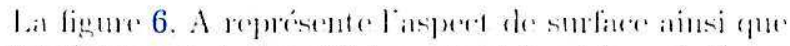

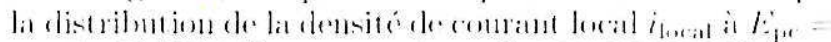

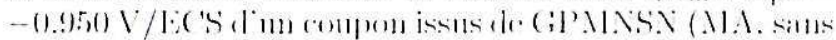

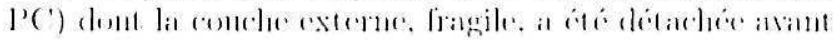
lissat. latissint ainsi mue surface reronterte dium soms(o)

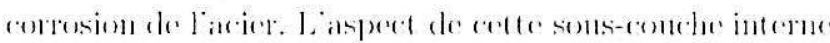

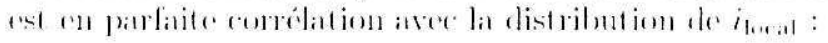

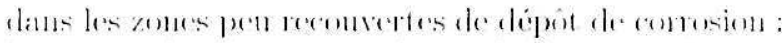

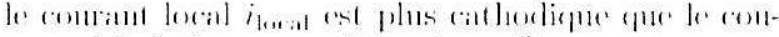

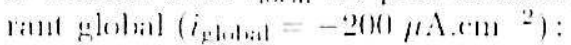

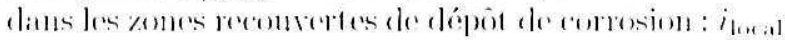

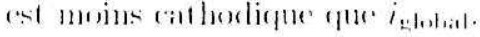




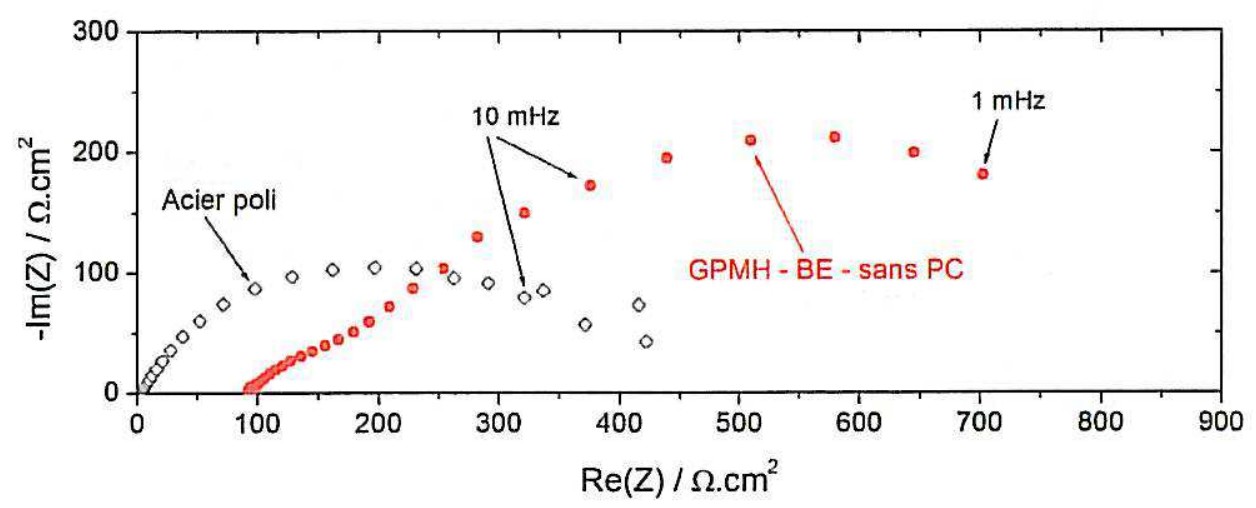

Fig. 7. Diagranumes d'impédance tracés en solution $\mathrm{NaCl} 0,5 \mathrm{~N}$ aérée sur la sufface d'acier polie et d'un coupon issus du GPMH (BE, salls PC).

Fig. 7. Impedance diagrams plotted in $0.5 \mathrm{M} \mathrm{NaCl}$ aczated solution, of the polished surface and of a coupon eaposed in site GPMII (BE without PC).

\subsubsection{Surfaces recouvertes de produits de corrosion et de matières organiques (catégorie 2)}

Lia figure 6, B représente la distrilution de ilocal d'um (о) interne contenant, en plus de produits de corrosion, des matières organiques (coppuillages). $\dot{A}$ la différence des surfares micpuement recouvertes de produits de cormosions. la distribution de la densité de comant lowal de l'échantillon ne comespond pas a lasperel de sa surface en fonction de la présence des rooguillages. 11 semble done gure la présence de corpuillage nünfluence pass la réactivité de lis conche de probluts de comosion sons-jacente.

\subsection{Spectroscopie d'impédance électrochimique}

\subsubsection{Diagrammes d'impédance type}

\subsubsection{Surface exempte de produits de corrosion}

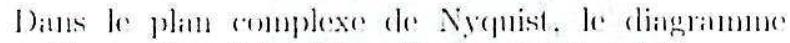

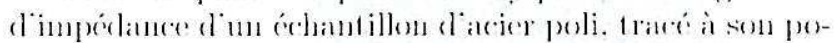

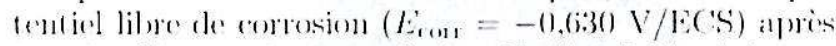

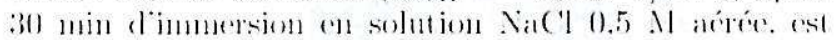

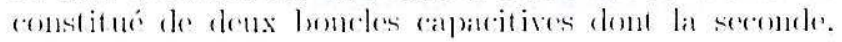

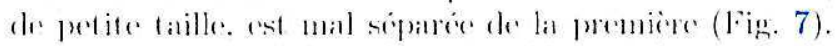

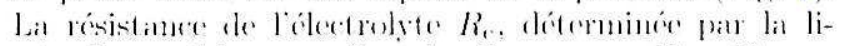

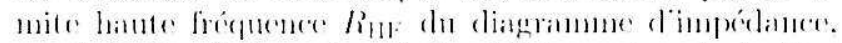

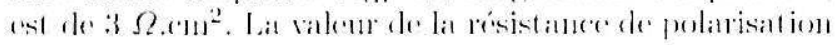

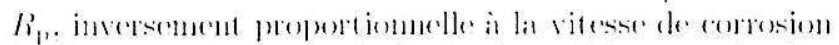

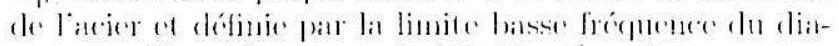

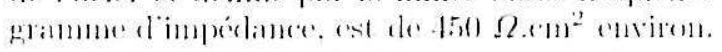

\subsubsection{Surface corrodée}

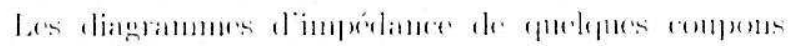

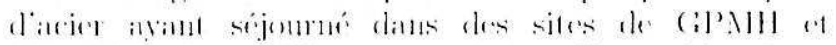

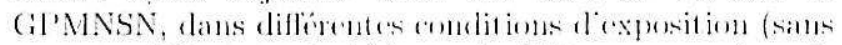

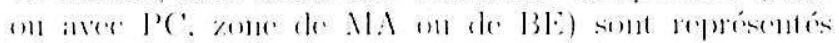
and figmes 7 of 8 . Dans foms lese rate lese diagrammons d"inpéclance sont constit nés de denx boncles capacitives comme cost le cas de la surface polie. ef présentent les raractéristicpues communes suivantés:

- me faible dinension de ba bonede hante frégnener:

- me augmentation importaute de la taille de la boucle basse frécuuence. Datus la plupart des cals. elle tend vers nue rloite de pente comprise entre 1 et 0.4 (angles correspondints entre $45^{\circ}$ et $\left.22.5^{\circ}\right)$.

En plus de la modification de labllure globale du diatgramme d limpédanee, la présence de dépests de corresion ì la surfare de lacier fait égalenent angmenter lompédanes du systome. Du cotó des latutes frépuences. la linite hav mugmente de 1 a 2 ordres de gromdere : R R des stor-

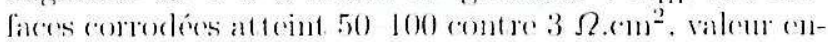

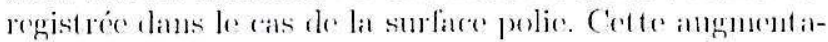

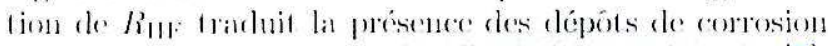

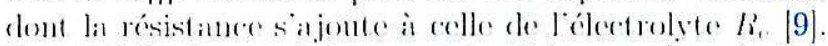

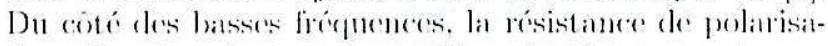

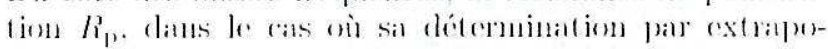

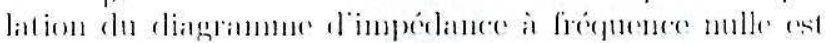

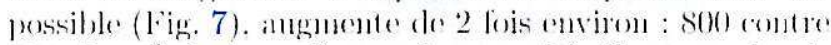

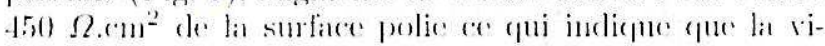

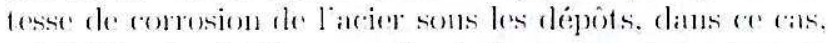

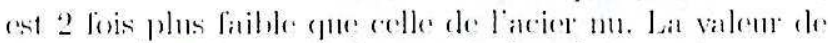

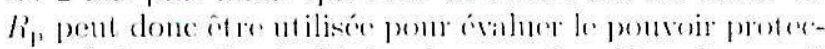

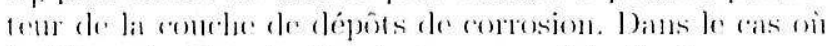

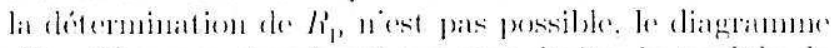

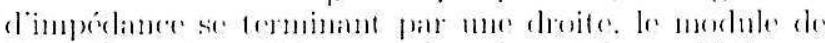

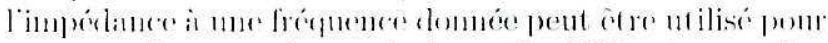

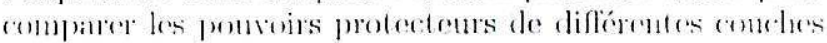

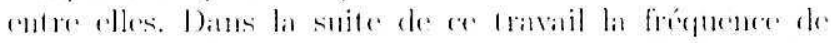

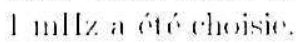

\subsubsection{Róle de la sous-couche interne}

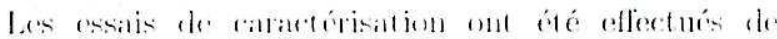

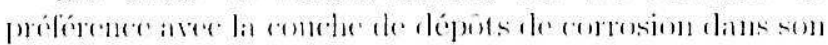

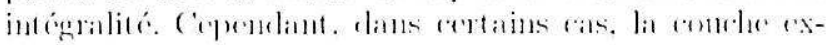

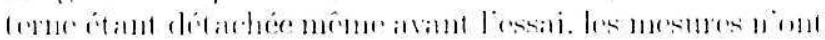




\section{A : GPMH}

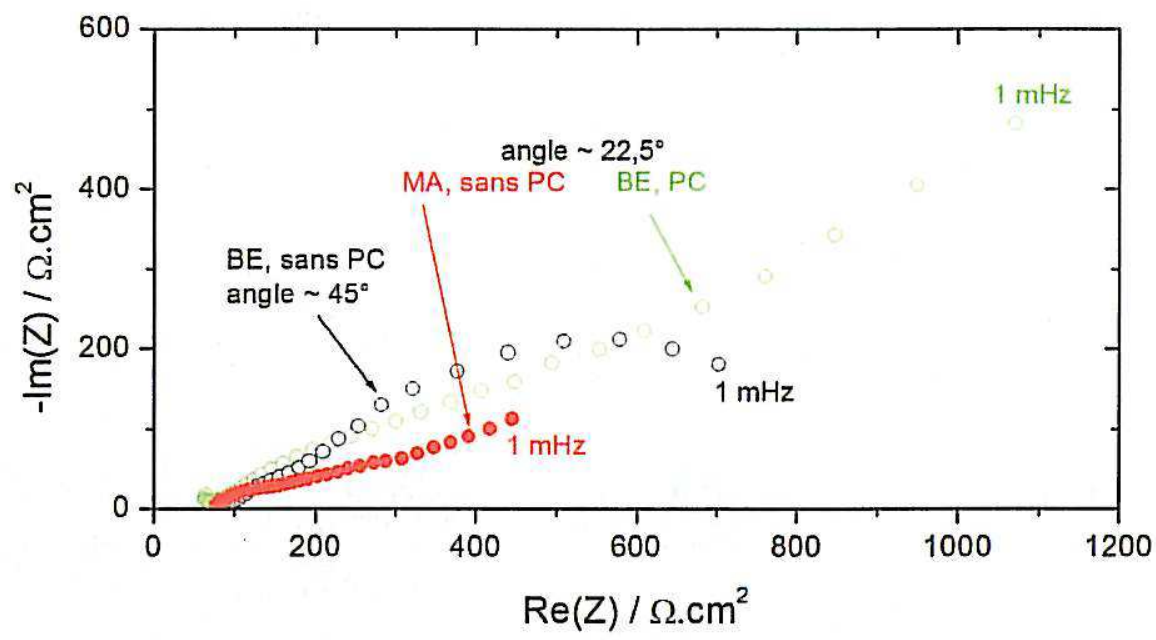

\section{B : GPMNSN}

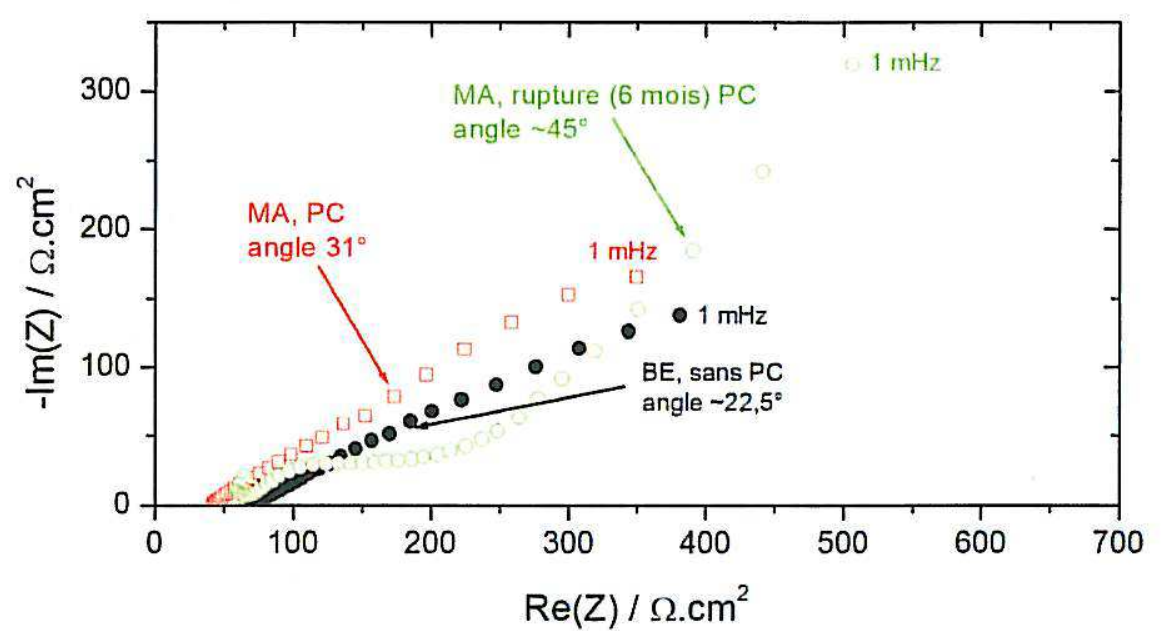

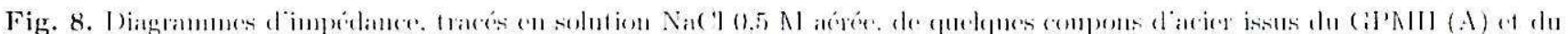
(IININST (13).

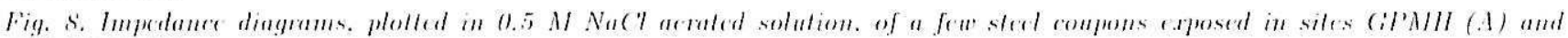
(iPMINSSN (B).

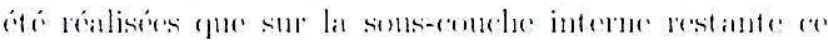
qui rend difficile la compariason des contelus forménes diuns

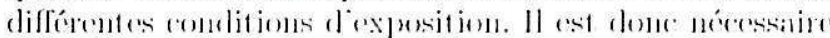

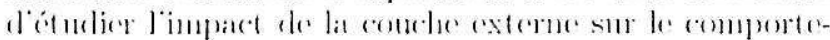

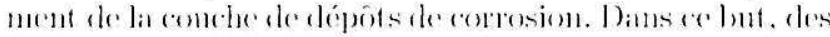

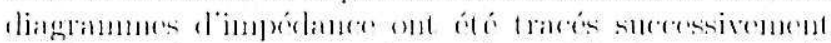

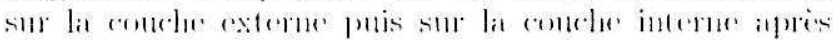

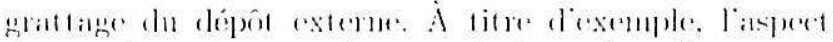

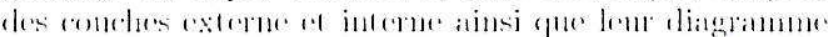

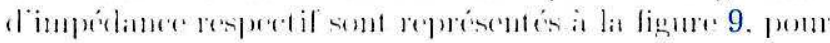

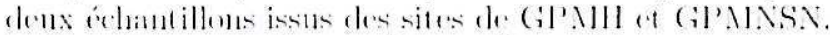

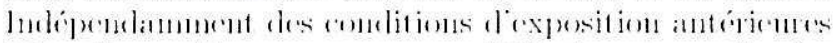

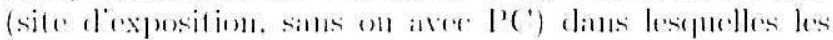

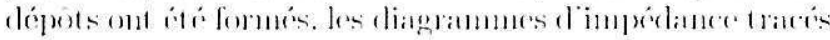

sur les deux couches externe et interne d'un mone rom-

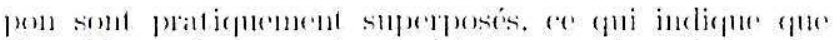

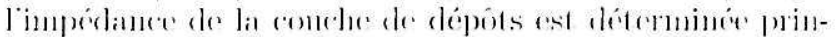
ripalement pall sal solks-comede interme.

\subsubsection{Influence des conditions d'exposition}

\subsubsection{Face d'exposition de coupon}

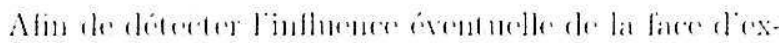

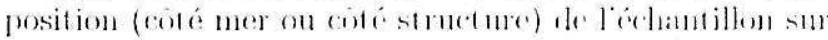

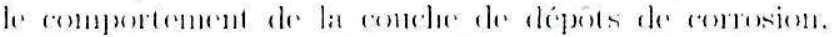

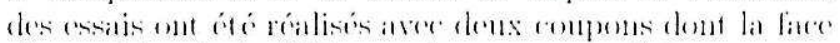

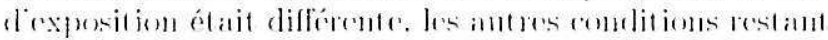



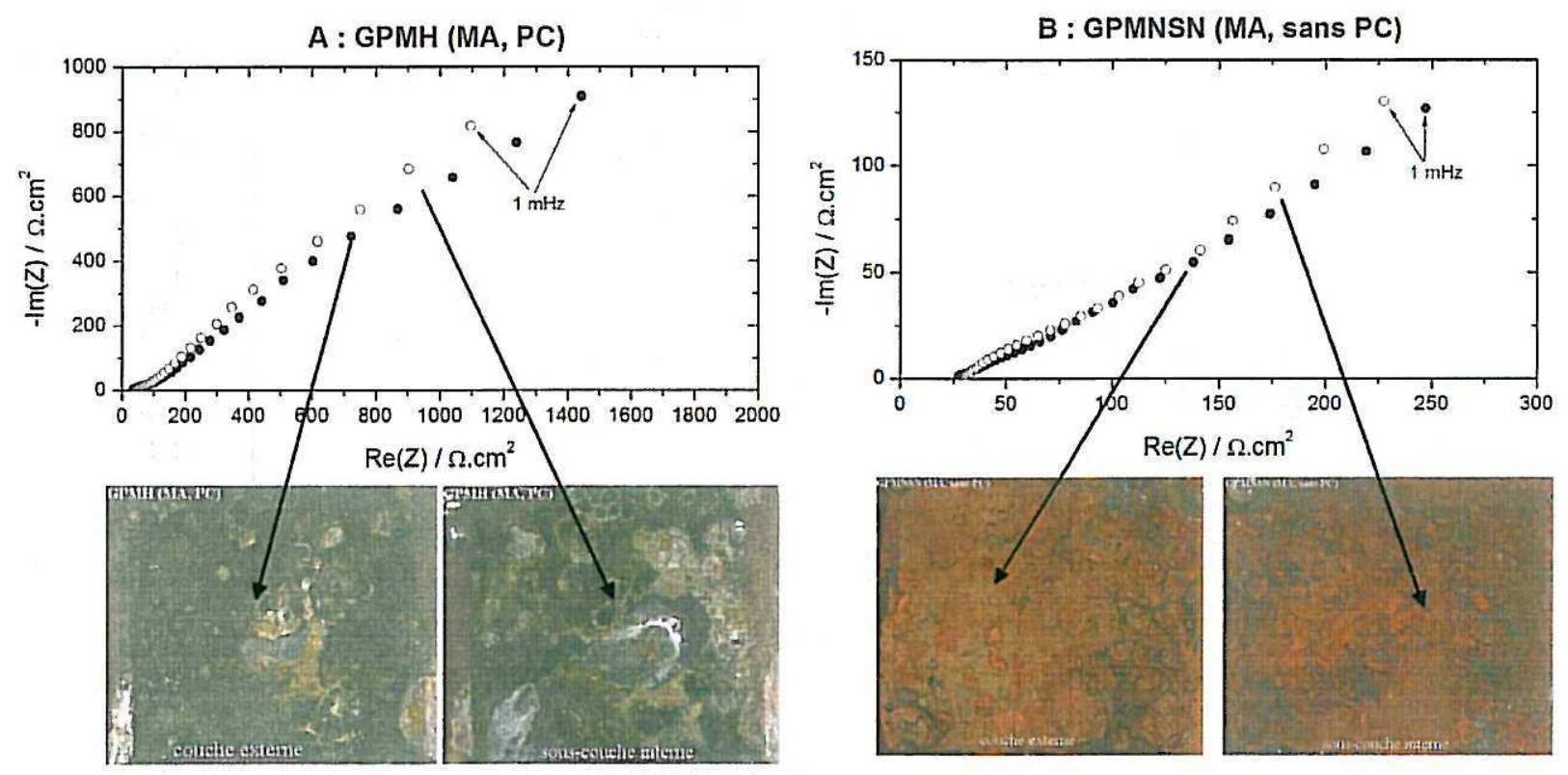

Fig. 9. Diagrammes dimpédance, tracés en solution NaCl $0,5 \mathrm{~N}$ ácée, des conches externe of interne des coupons issus du (EPMH (A) et du GPNNSN (B).

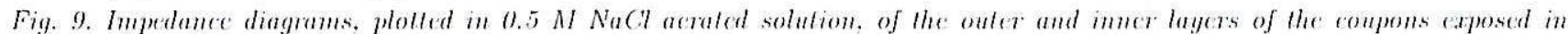
siles GPMII (A) and GPMINSN (B).

identiques. Liaspect des faces coté mer ef coté struk-

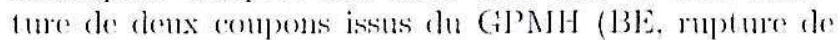

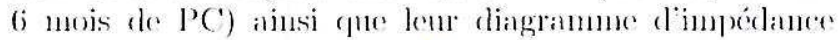
sont représentés ì lat figure 10. Malgré la différenere dians

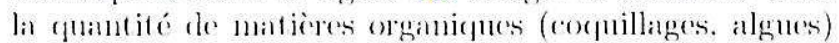
présente dauns aes denx cometues de dépots. les diagrammues

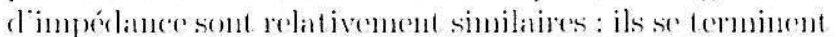

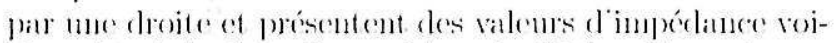

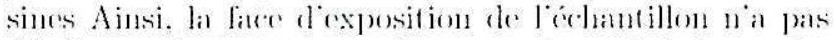

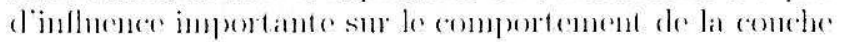
de dipots de comosion formose.

\subsubsection{Protection cathodique}

\section{- Avec ou sans PC}

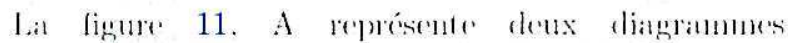

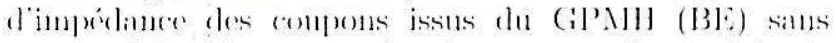

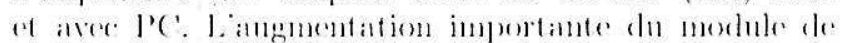

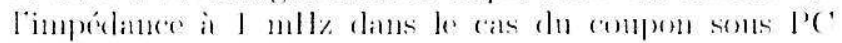

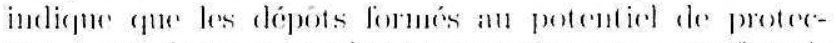

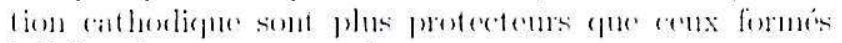

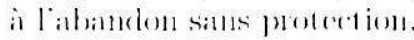

\section{- Influence diune interruption de PC}

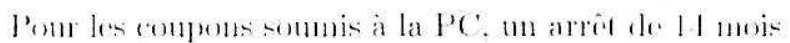

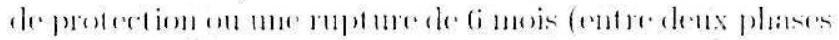

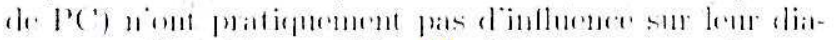

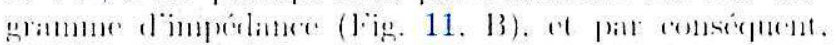

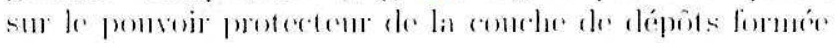
inilinloment sols l'(')

\section{- Influence d'une application différée de la PC}

Il fint noter que la mise sous PC: (penclant of 14 mois) d'élantillons init balenent non protégés n affecte pas leur

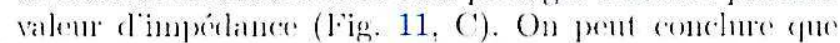
dans ce cas, la mise sous PC diflérée 11 mólione pas lo

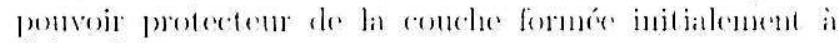
liabaluclens.

\section{Discussion}

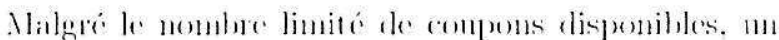
seul (o)

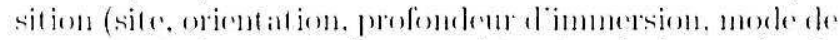

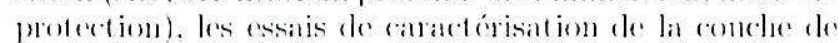

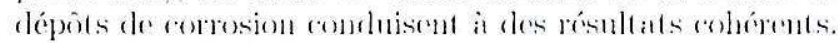

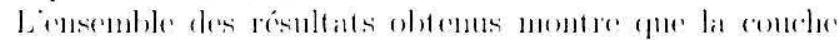

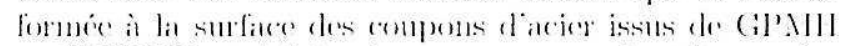

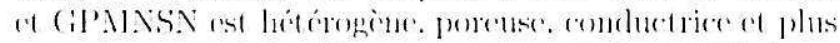

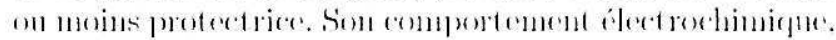

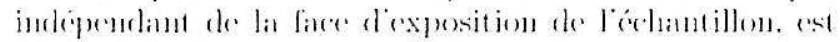
détermine par sia soms-comehe inderme.

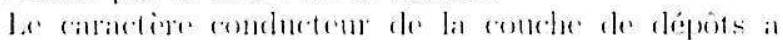

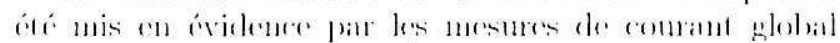

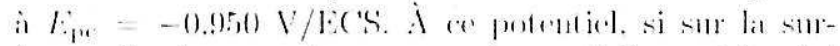

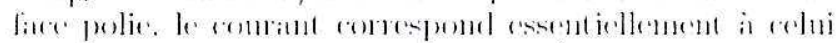

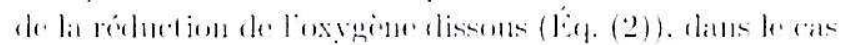

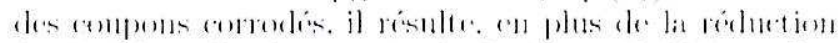

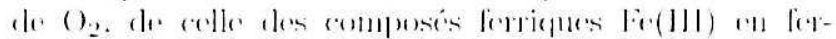

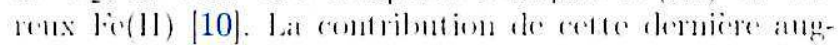
mente ane le ponvoir oxpelant do milien a dexpestion de

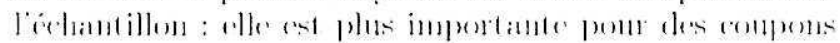




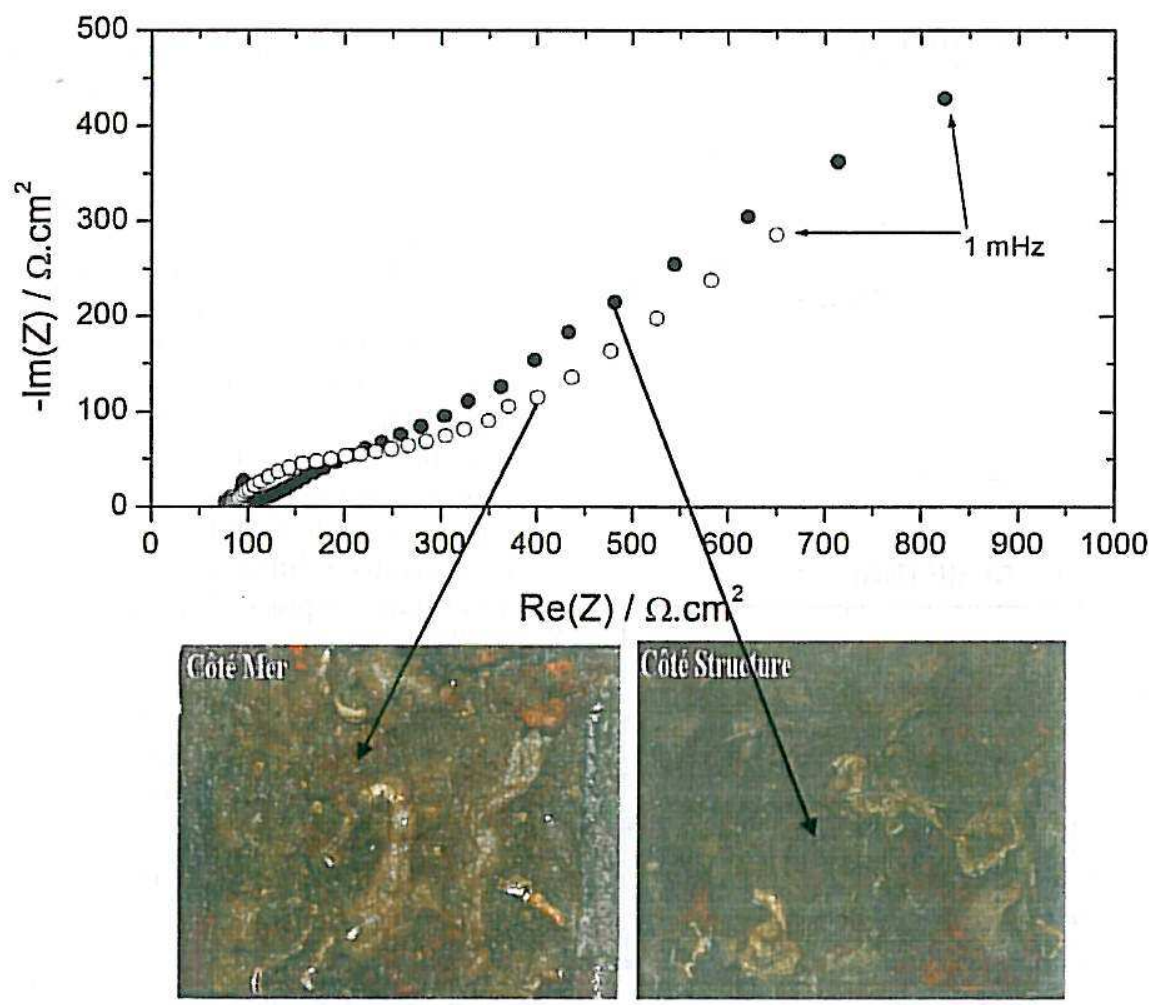

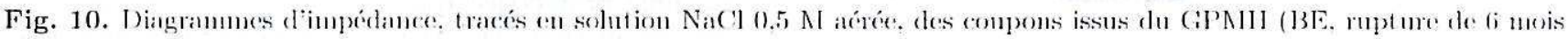
de P') exposés côté mer ou côté strueture.

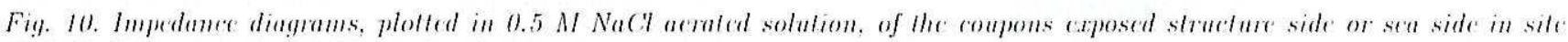
(iPMII (BE. o months of P': broling).

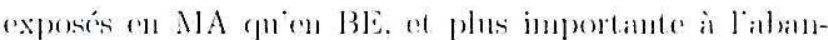

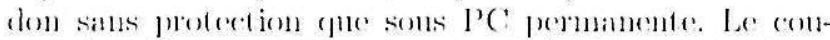

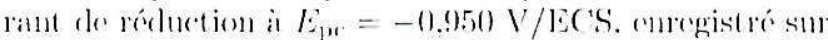

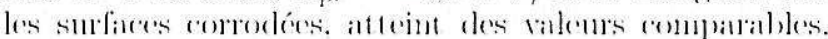

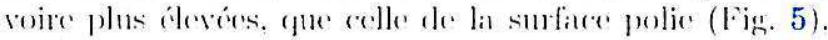

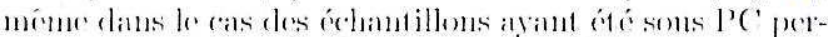

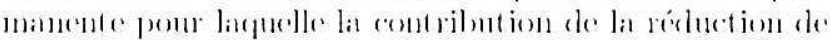

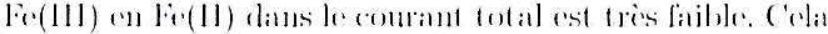

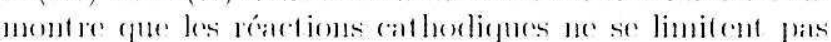

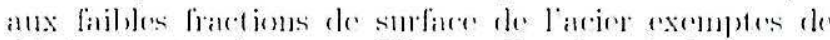

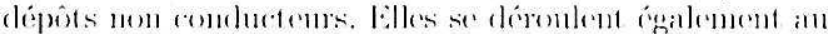
sein de la romele de dépots de corrosion qui doit intre

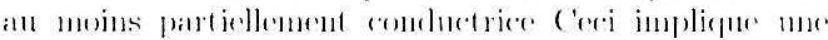

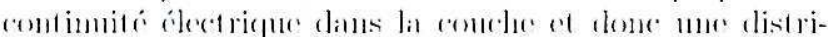

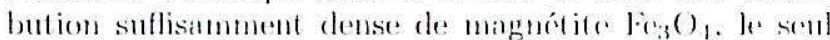

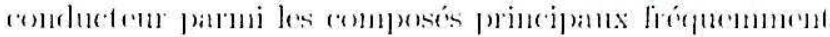

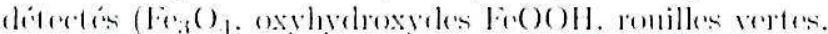

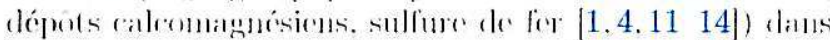

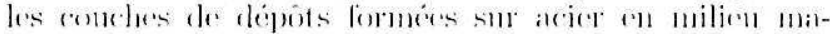

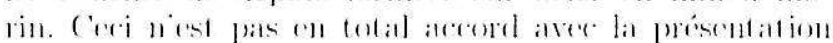

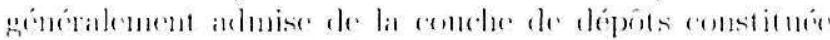

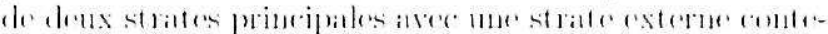

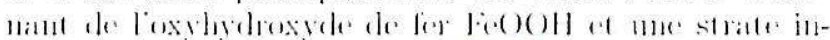

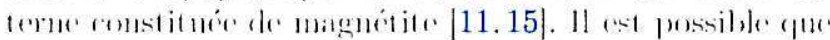

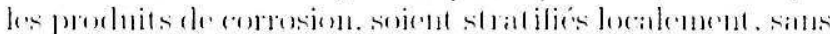

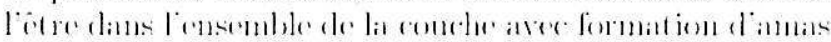

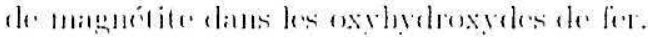

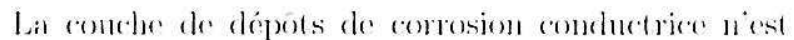

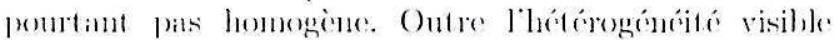

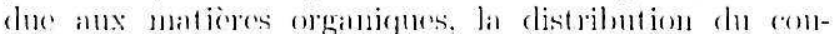

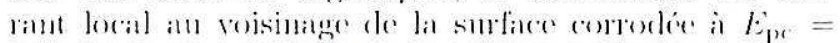

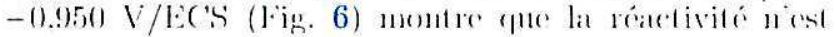

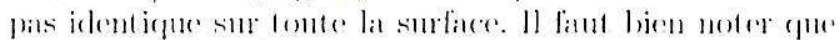

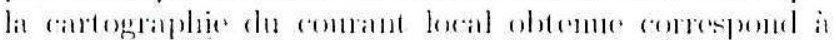

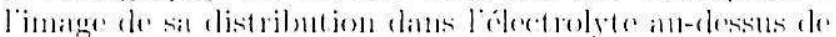
lit surfice mais pats a celle de la surface elle-mene qui ne

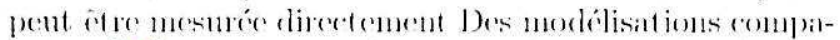

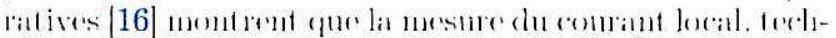
niqgue mise au point par lafle et Nuccitelli [17] pour des

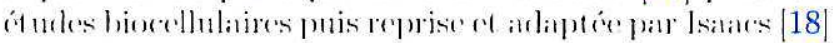

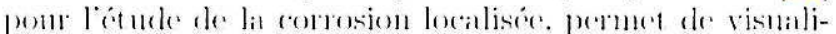

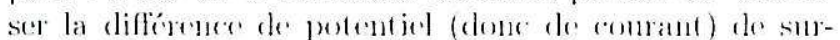

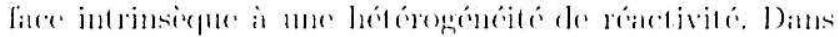

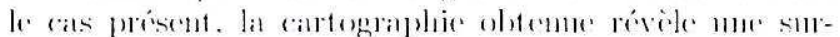

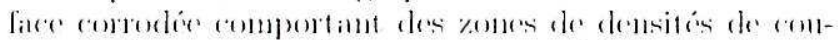

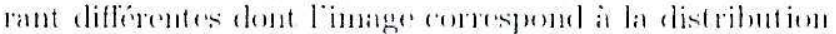

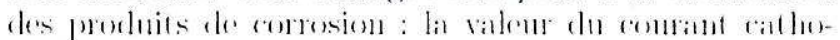

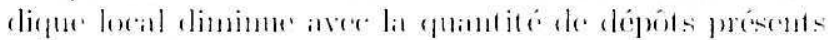

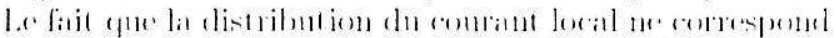

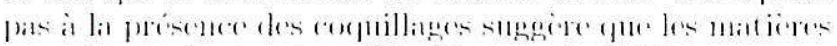
organiques nont qu'un effet d'érin tris lialles.

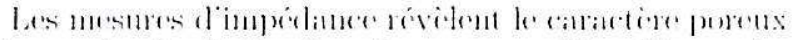

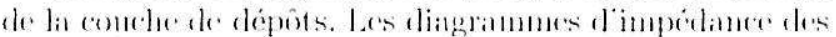

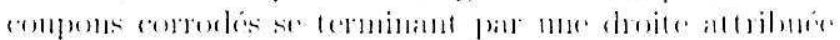



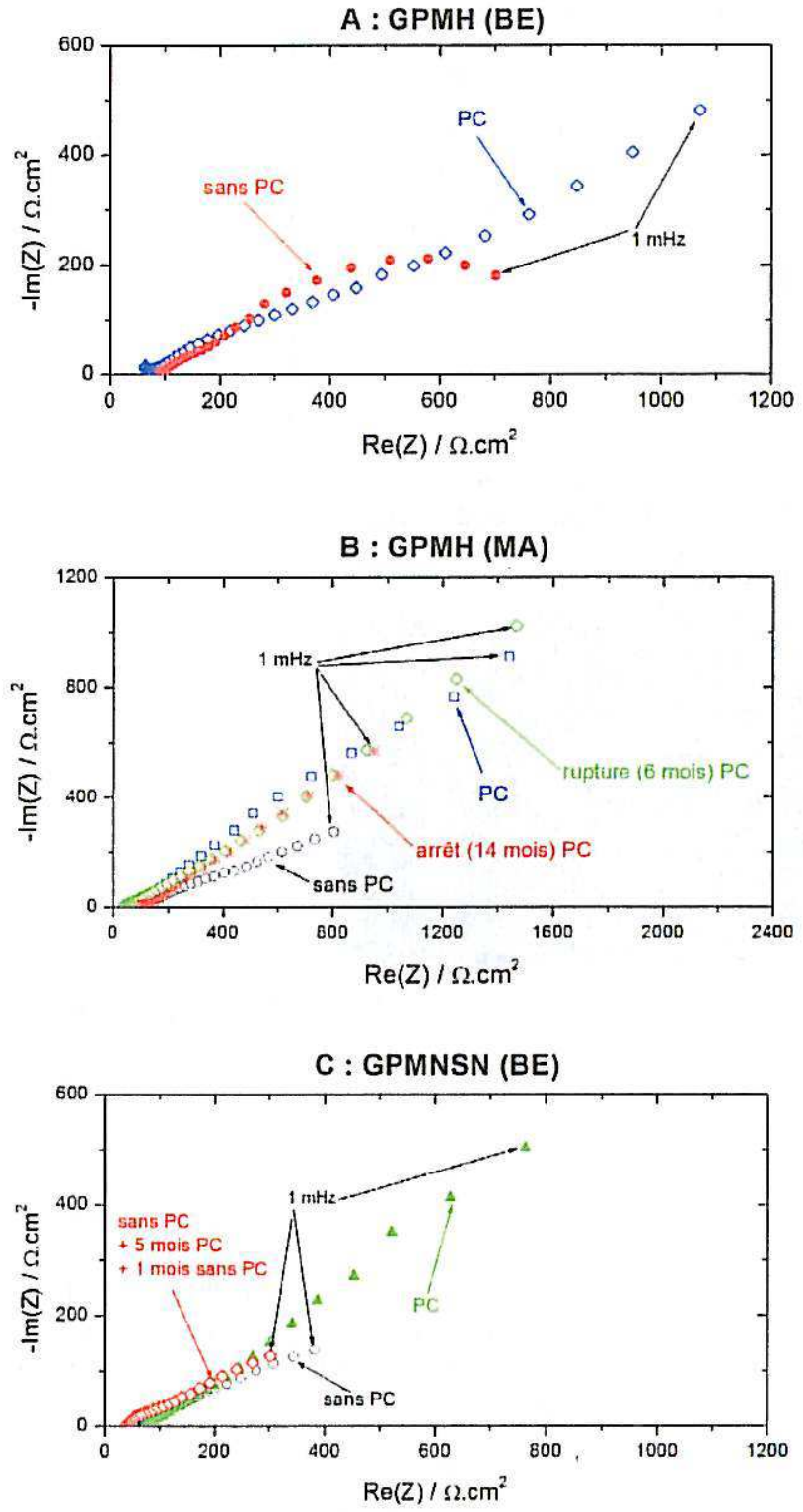

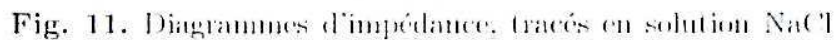

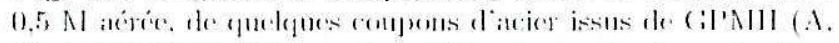

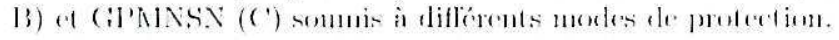

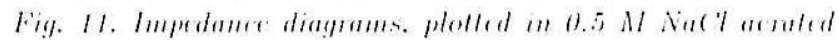

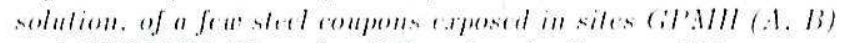
and CiPMNSN (C') under differenl protection conditions.

lablifuellenent is la diffusion (Figs. 7. 8) Mamluisent lien

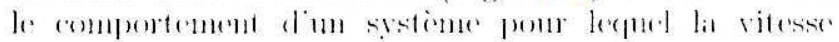

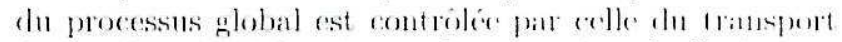

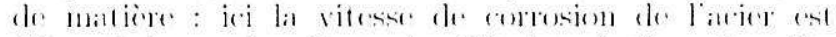
deternine par la vilesse de diffusion de l'oxygine dis-

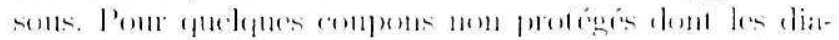

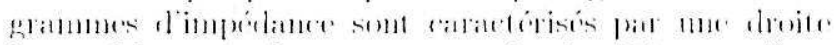

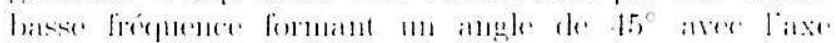

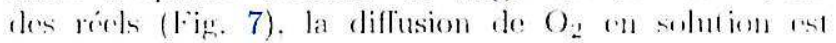

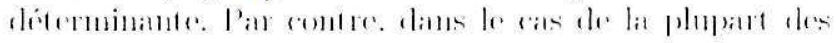

coupons pour lesquels la droite de diffusion forme un angle de $22,5^{\circ}$ avec l'axe des réels, il faut considérer la diffusion de $\mathrm{O}_{2}$ dans les pores de la couche de dépots conductrice. Ce résultat est conforme à la théorie de De levie, concernant l'impédance de diffusion de $\mathrm{O}_{2}$ dans les pores cylindriques de longueur semi - infinie d'une électrode porense [19.20]. Ainsi, les diagrammes d"impédance des compons montrent dime part la texture poreuse de la couche de dépots of dautre part, ils confirment son caractère conducteur déjà mis en évidence par des mesures du comant is $E_{\mathrm{pc}}=-0,950 \mathrm{~V} /$ ECS. Les valeurs intermédiaires (entre $45^{\circ}$ et $22,5^{\circ}$ ) de l'angle entre la droite basse fréquence et l'axe des réels $\operatorname{Re}(\mathrm{Z})$ indiquent le caractère mixte de la diffusion de l'oxygène dissous à la surface corroclée : diffusion en solution sur une partie de surface et dans les pores de la couche de produits de comrosion sur d'autres.

Les conches de dépôts de corrosion possèdent un cretain pouvoir protedeur. En présence dime conche de dépôts, lacier se corrode plus lentenuent. Sa vitesse de corrosion est divisée par deux en présence des dépôts mêne les moins protecteurs (Fig. 7). Le pouvoir protecteur de la couche est déterminé principalement par celui de sa sons-conche interne. 11 dépend done des conditions de formation de cette derniere. Ainsi, la conche formón an potentiel de protection cathoclicuee $E_{\mathrm{pu}}=-0.950 \mathrm{~V} / \mathrm{ECS}$

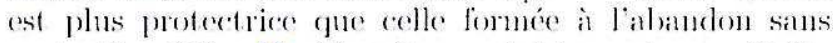
protection (Fig. 11, A) grâce probablenent anx clépôts catcomagnésiens dont la formation est plus forvomble is (e potentiel sule is liangmentation do pH interfacial

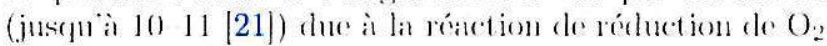

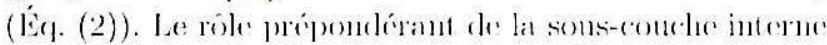

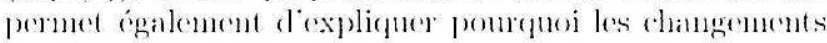
du mode de protection naflectent matiquennent pas k

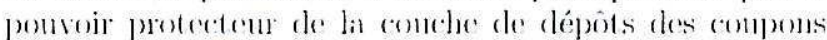

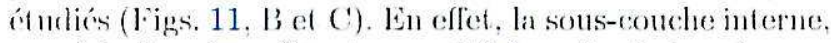

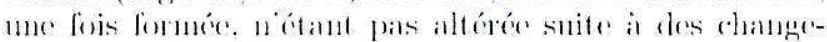

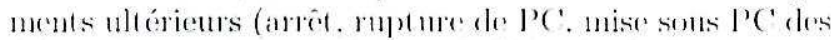

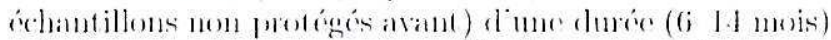

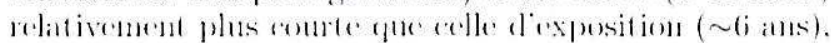

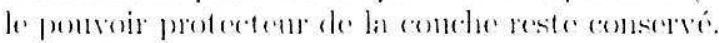

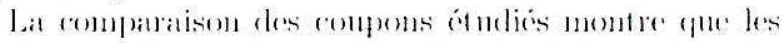

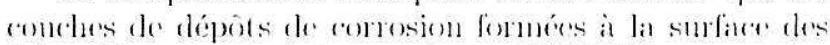

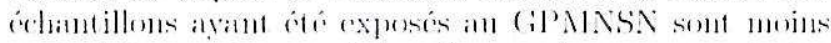

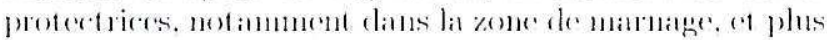

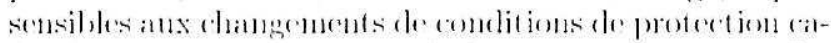

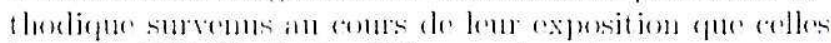

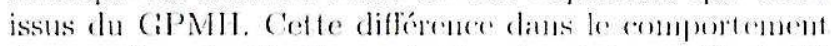

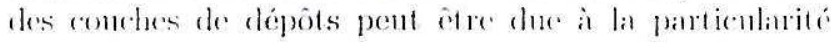

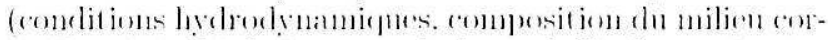

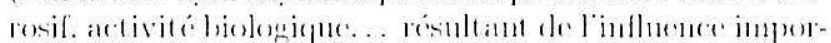

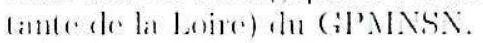

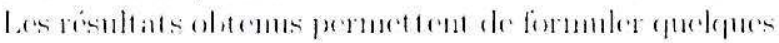

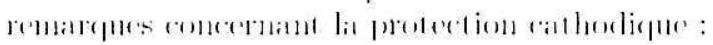

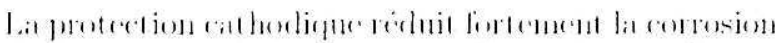

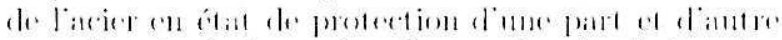

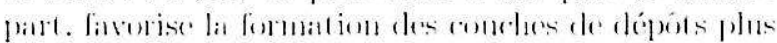

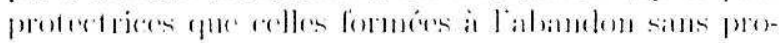

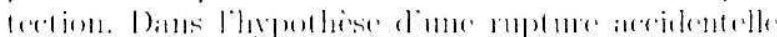


de PC, l'acier initialement protégé se corrode, dans les premiers instants après la rupture de PC, beancoup plus lentement que celui non protégé. Un arrêt relativement court (de quelques mois) de PC n'a pas de conséquences dramatiques.

- Il est soulaaitable que la PC soit appliquée dies le clébut de l'exploitation de l'ouvrage. L'application différée de $P C$ après une période sans protection d'une part ne permet pas d'améliorer le pouvoir protecteur de la conche de dépôts formée initialement à l'abandon et d'autre part, entran̂e me angmentation du besoin en courant à cause de la réduction électrochinnique simultanée d'une quantité inportante de composés ferriques présents dans cette conche.

- A $E_{p c}$, la conche de dépots se comportant comme une callhode poreuse à grande surface développée, le comrant pent atteindre des valeurs importantes et, contrairement a liolée généralement admise, il pent ne pas diminuer avec le temps. Ainsi, une sons-est imat ion du besoin en coumant, notanment en comant de maintien de la polarisation, conchurait is des potentiels insullisants pour whe protection eflicace.

\section{Conclusion}

Les propriétés cólectrochimiques de la comche de dépots

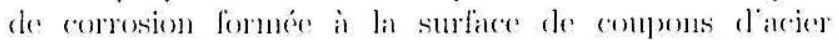
E2t immorgés pendant plusiours anmés stur des siles des Cirands lorts Maritines du Havre at de Nantes Sanit-Nazaire sous différentes comblitions de protection

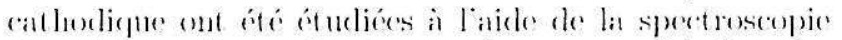

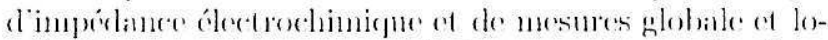

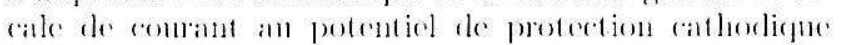
$E_{11}=-0.950 \mathrm{~V} / \mathrm{EC}(\mathrm{S}$.

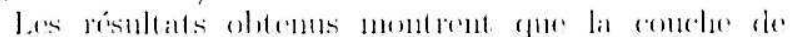
dépots dont le (om)

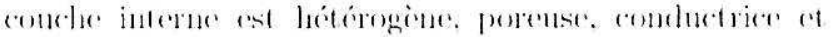

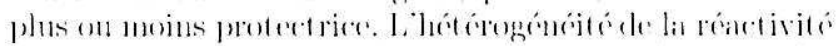

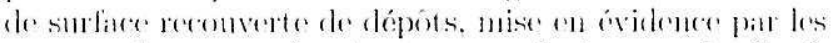

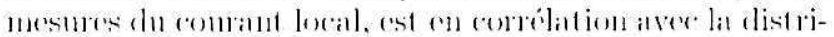

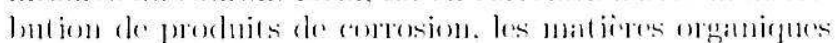

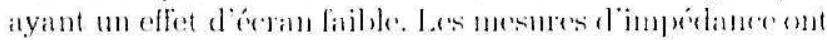

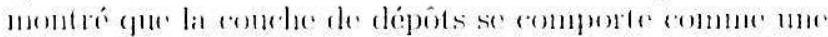

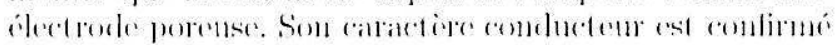

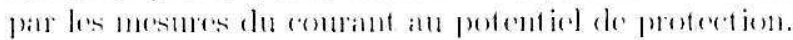

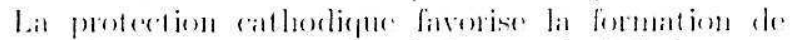

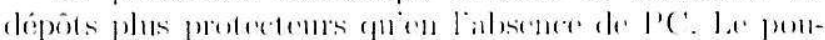

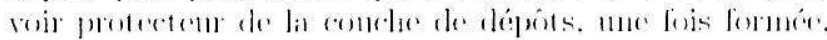

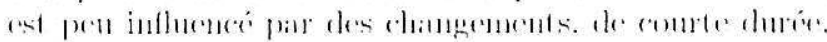

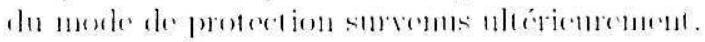

Remerciements. Les anteurs renercient Vincent Vivier du LISE pour son aide dans la réalisation des mesures du couriant local.

\section{Références}

[1] T. Misawa, K. Asami, K. Hashinoto, S. Shimodaira, Corros. Sci. 14 (197-1) 131-1.19

[2] R.M. Comell, U. Schwertmanu, The Iron Oxides: Structure, Properties, Reactions, Occurrences and Uses. 2ud edu., Wiley-VCH, 2003

[3] L.L. Shreir, R.A. Jarnan. (i.T. Burstein (Eds.). Corrosion, vol. 1. Metal/Enviromment Reactions. 3rd edn., Butterworth-Heinemann, Oxford, 1994. $3: 1-3.18$

[1] J.B. Nemet, La corrosion marine des structures métalliques portutires : études des mécanismes d'amorçage et de croissance des produits de corrosion. Thèse de doctorat. Université de la Rochelle. La Rochelle. France, 2000

[5] R.E. Molchers, Comos. Sci. 45 (201:3) 923-940

[6] R.E. Melchers, R. Jeffrey, Comos. Sri. 47 (2005) $1678-1693$

[7] Y.F. Wen, (:Z. C'ai, X.H, Liu, J.F. Pei, X... Zlu, 'T.'T. Xiase. Comos. Siri. 51 (2009) 319(9-355

[8] R.E. Nelchers, Corros. Sci. 68 (2013) 186-19.1

[9] L. Bonsselni. ('. Finud, B. Tribollet. E. Triki.

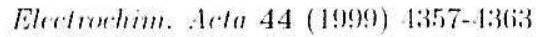

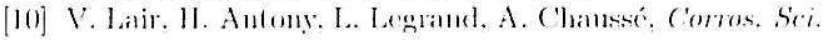
48 (20)(6) $2(15)-20(6 i 3$

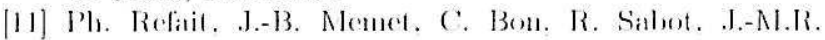
Ginin. Comos. Sir. 45 (20013) $8333-8.15$

[12] S. Ellocik. A.C.C. Tisemun. A.L. Narkay. ('omos. Sri. 26 (I)

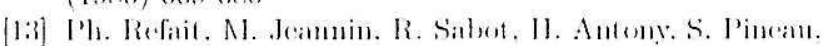
('orrous sici. 71 (20113) :32-36;

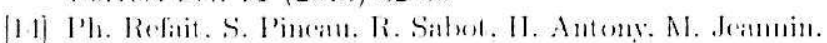

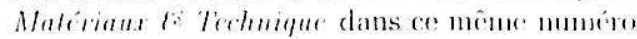

[15] (i. Beranger. H. Narzille. ('orrosion des metaux of at-

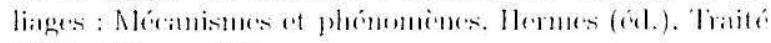
MINI. 20002. p. 36ir

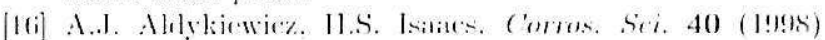
$\mid(i ;-i-16 i \mid 6$

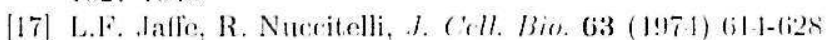

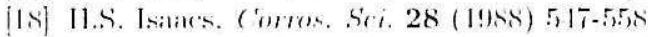

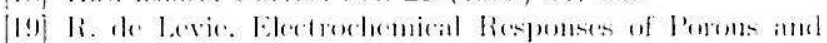

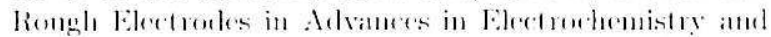

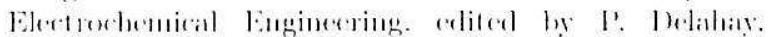

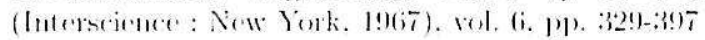

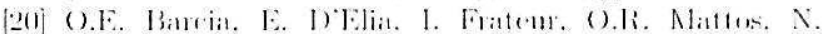

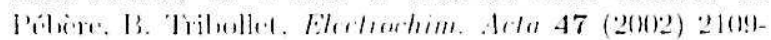
2110

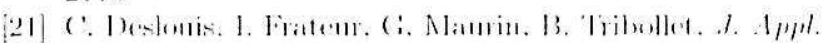

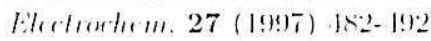

